\title{
Intelligent Technique for Seamless Vertical Handover in Vehicular Networks
}

\author{
Shidrokh Goudarzi ${ }^{1}$, Wan Haslina Hassan ${ }^{1}$, Mohammad Hossein Anisi ${ }^{2}$, Muhammad \\ Khurram $\mathrm{Khan}^{3}$, and S.A. Soleymani ${ }^{4}$ \\ ${ }^{1}$ Communication System and Network (iKohza) Research Group, Malaysia-Japan International Institute of \\ Technology (MJIIT), Universiti Teknologi Malaysia, Jalan Semarak, 54100 Kuala Lumpur, Malaysia. \\ shidrokhgoudarzi@gmail.com,wanhaslina@ic.utm.my \\ ${ }^{2 *}$ School of Computer Science and Electronic Engineering, University of Essex, Colchester, CO4 3SQ, \\ United Kingdom.m.anisi@essex.ac.uk \\ ${ }^{3}$ Center of Excellence in Information Assurance, King Saud University, Riyadh 11451, Saudi Arabia. \\ mkhurram@ksu.edu.sa \\ ${ }^{4}$ Department of Computing, Faculty of Computing, University of Teknologi Malaysia, Johor Bahru, \\ Malaysia. asseyed4@live.utm.my
}

\section{Abstract}

Seamless mobility is a challenging issue in the area of research of vehicular networks that are supportive of various applications dealing with the intelligent transportation system (ITS). The conventional mobility management plans for the Internet and the mobile ad hoc network (MANET) is unable to address the needs of the vehicular network and there is severe performance degradation because of the vehicular networks' unique characters such as high mobility. Thus, vehicular networks require seamless mobility designs that especially developed for them. This research provides an intelligent algorithm in providing seamless mobility using the media independent handover, MIH (IEEE 802.21), over heterogeneous networks with different access technologies such as Worldwide Interoperability for Microwave Access (WiMAX), Wireless Fidelity (Wi-Fi), as well as the Universal Mobile Telecommunications System (UMTS) for improving the quality of service (QoS) of the mobile services in the vehicular networks. The proposed algorithm is a hybrid model which merges the biogeographybased optimization or BBO with the Markov chain. The findings of this research show that our method within the given scenario can meet the requirements of the application as well as the preferences of the users.

Keywords: Heterogeneous Networks, IEEE 802.21, Vertical Handover, Markov chain, Biogeography-based optimization, Vehicular Network (VN) 


\section{Introduction}

Based on the idea of Internet of Things (IoT) [1-4], different kinds of vehicles and devices would be able to communicate with each other through various communication technologies. Therefore, current vehicle equipped with mobile routers or nodes can have multiple interfaces and access to various wireless networks such as WiFi, WiMAX, and 3G. Heterogeneous networks used for seamless mobility will face prominent problems in mobile IP networks in the future. This is because there are different factors, which would significantly affect the optimized handover among the various technologies used for accessing the network, such as the vertical handover (VHO). Some of these factors are congestion, load, strength of the signals, bandwidth, connection stability, battery life, as well as other factors that are temporal and spatial. A mobile user in the heterogeneous wireless networks might have to carry out handovers over various domains of network to sustain the connection of data and the QoS. The VHO process includes 3 stages including the information gathering, decision-making, as well as the handover execution. The information that is acquired is utilized to identify the present and most suitable networks for the specific application in the following stage known as the stage of handover decision-making.

A vehicle within the vehicular networks (VNs) is regarded as a network node that is equipped with many interfaces offering access to various technologies including $\mathrm{Wi}-\mathrm{Fi}$, GPS, WiMAX, Long-Term Evolution (LTE), and UMTS. The vehicles involved can communicate with each other and with the point of attachment (PoA) including the base stations (BSs) or the access points (APs) using the infrastructure or the ad hoc modes [5,6], accordingly. The contexts of the vehicular systems based on a wireless communication perspective is highly robust and vehicles should be equipped to manage the heterogeneity using capabilities of awareness of context and VHO. To establish the awareness of context, the vehicles, as well as the other networking components such as the APs or the BSs, must provide beneficial information regarding the network status, geo-locations, and the assets of the network provider including their specifications. In addition, besides providing information regarding the capabilities of the technologies, the vehicles should also offer suitable information about the preferences of the users. About the VHO, the network's components should be able to combine the standard primitives 
of IEEE 802.21 [7] to allow the features of the protocol of the MIH function to offer a homogeneous handover interface that is seamless within the various heterogeneous wireless networks such as WiMAX, Wi-Fi, LTE, and UMTS. Moreover, it is important to perform a decision-making process to select the most appropriate correspondent node (CN) based on the set of heterogeneous wireless access networks that are available. This should be done by taking into account the various contextual factors and the ways in which the various networks perform. It is imperative that this procedure is precise and quick in order to prevent affecting the QoE or the connectivity negatively.

Several challenges are present in the multi-hop networks that are wireless [8-11] as well as in the decision stage of the vertical handover while the procedure for handover is going on. The main problem in providing seamless vertical handover (VHO) is maintaining the required mobile Quality of Service (mQoS) across different access networks with multiple resources in neighboring heterogeneous networks. The challenges related to the handover process such as packet loss, high latency, and signaling cost (typical in horizontal handovers), are further complicated given the complexity and delay across different access network technologies. Thus, there is a need to develop an effective algorithm for vertical handover decision-making (VHD) that would be able to choose the best-optimized access network for the handover process while maintaining the stability of this connection throughout the session. This would entail complex calculations in measuring the VHD algorithms from a multitude of parameters. One method to accomplish this requirement is using intelligent algorithms that can adapt and optimized the VHD problem effectively and provide the most optimal network selection. Hence, the research question of this study may be stated as follows:

" How might an adaptive algorithm be developed to support effective and seamless vertical handovers for heterogeneous networks without incurring high costs in complexity?"

Towards this end, this study proposes a novel hybrid algorithm for vertical handover decision or VHD using two major approaches namely the bio-geographical based optimization or BBO method and the Markov chain method for vehicular networks based on the category of the infrastructure mode (i.e., communications based on AP instead of the conventional ad hoc approach or the vehicular ad hoc networks or VANETs). The 
experiment investigates the effect of migration model on $\mathrm{BBO}$ performance using the Markov chain model. In $\mathrm{M}-\mathrm{BBO}$, each state describes how many individuals at each point of the search-space are there in the population. Probability Pij is the probability that the population transitions from the ith population distribution to the $\mathrm{jth}$ population distribution in one generation. M-BBO considers the immigration of each solution feature as separate probabilistic trials. Recall that evolutionary algorithms (EAs) use fitness values to perform the basis of selection. However, the probability distribution instead of fitness values for selection has been used in our proposed Markov chain-based selection uses. Our idea is incorporated in population proportion-based selection by approximating the probability distribution of the population sizes and then performing selection based on approximate distribution. This idea would merge the advantages of (EAs) with the advantages of probability distribution based selection. The proposed VHD uses the commission of the standard of IEEE 802.21. Figure 1 demonstrates the specific journey of the vehicular network in an urban setting along with a heterogeneous wireless access coverage with various corresponding ranges.

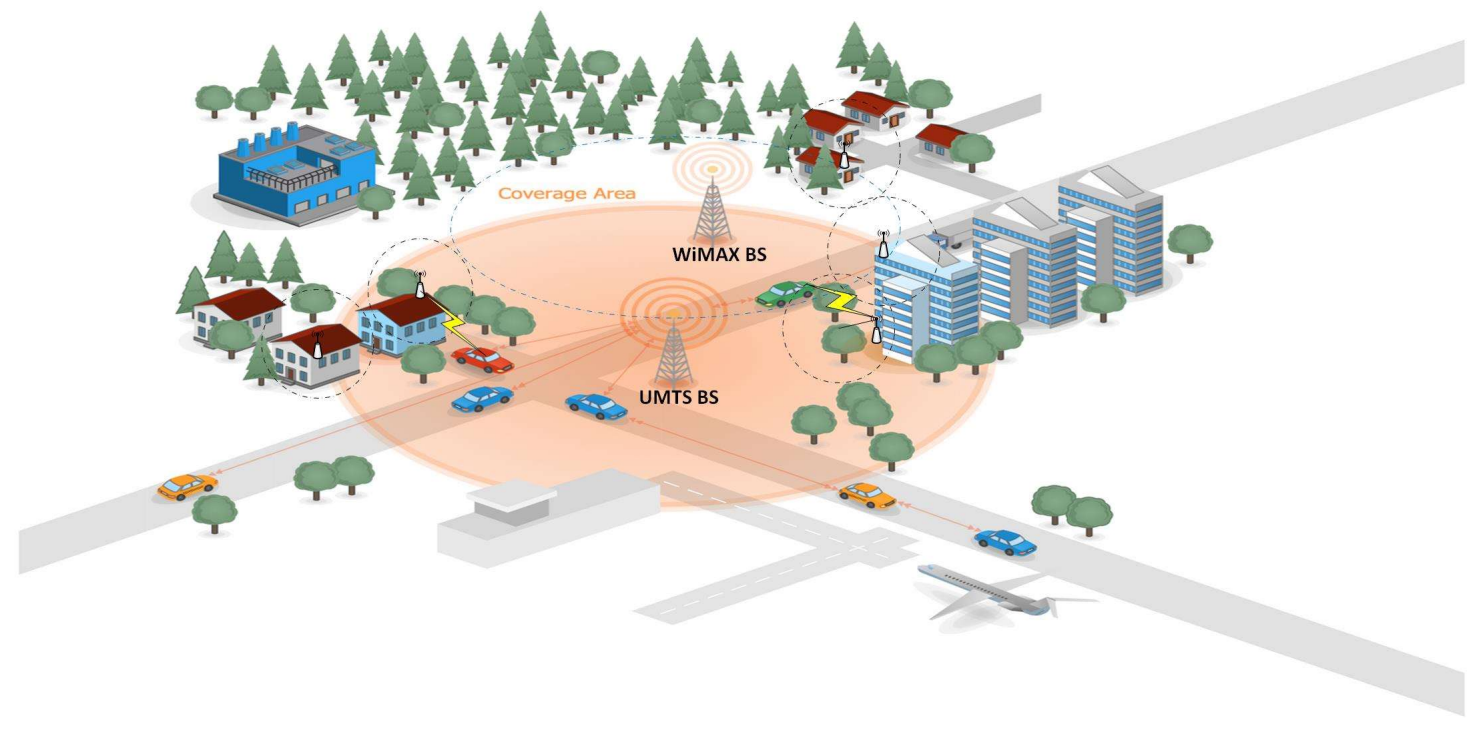

Figure 1. Heterogeneous wireless networks.

This study is organized into several sections namely: Related literature review is carried out in Section 2 while the following section explains the network model. VHO as the optimization problem is formulated in Section 4 while Section 5 provides the developed 
solution. Section 6 discusses the outcomes of the simulation while Section 7 presents the conclusion of this research.

\section{Related Work}

Mobility management, which originates from the cellular networks, is a critical and problematic area in the support of a seamless communication. The location management and handoff management encompass the issue of mobility management. The location management includes the tasks of tracking and updating the present location of a mobile node (MN), while the handoff management is directed at maintaining the active connections when the MN shifts from its attachment point [12].

Mobility management is significant in offering a high-speed and seamless service for vehicular network as the MNs tends to shift their attachment points often, and the network topology can be abruptly shifted as well. Given the variations of the communications of V2I and V2V, their schemes for mobility management is developed in a different manner to reach an optimized performance. As the communication of the V21 requires Internet-based data exchange, for the purpose of interoperability and compatibility, many of the solutions for mobility management for communication of the V21 are developed using the Internet protocols for mobility management such as the Mobile IPv6. Mobility management for the communication of V2V largely emphasizes the discovery of the route, maintenance, as well as recovery, not unlike that found in the MANETs [13].

New findings were made by Petrut et al. [14], who found out that by utilizing measured cell quality value (RSRQ) as a handover parameter in heterogeneous networks, it is possible to gain improvements in achieved throughput and to reduce user equipment (UE) power consumption through lowered transmit power requirements.

In a dynamic scenario, a problem closely related to user association is the handover problem. Deciding on when to trigger a re-association is an equally important problem, and understandably has gained significant attention [15]. In the past decade as well, vehicular communication has been enhanced to include communication devices of short and long distances, the GPS, as well as vehicle sensing systems. The capabilities in communication utilize an extremely robust vehicular environment. Using GPS 
information to enhance the process of handover and the selection of network, within the parameter of a single wireless network, has been studied widely [16].

Information on geolocation could also be used to improve the process of decisionmaking for handover across heterogeneous networks. The study by Ylianttila et al. [17] established the first method of utilizing the GPS to manage the mobile device's present location. The proposal in this study took into consideration the scenario of the handover under the WiFi as well as the UMTS cells. The researchers considered the CN's received signal strength (RSS) in the process of decision making. The information from the GPS such as the coordinates, direction, and speed had been used by several researchers to improve the prediction of mobility and to enhance the VHO process through the path prediction and using it to find out the following most likely PoA in that path [18]. The authors of [19] take the mobility classes into account, but they do not differentiate between local and global HO problems and consider only the global HO parameters. The study by Wang et al. [20] proposed a VHO approach, which utilized certain factors including the data rate, RSS, the trend of movement, and the bit error rate (BER) that enabled the selection of the best-suited network along with the parameter of the prioritized decisions. The decision tree is utilized in this approach according to the selected parameter at each node of decision-making process, where it could stop or continue at that point accordingly. Moreover, this approach takes into consideration the underlying connecting different technologies such as IEEE $802.11 \mathrm{p}, 3 \mathrm{G}$, or WiMAX.

Nevertheless, the IEEE 802.21 is not considered as being a part of the VHO framework by this solution and deploys a solution that is customized to communicate with various network interfaces and entities. The research by Wang et al. [21] regarded the WiMAX and WiFi as one of the components of the underlying connection. They consider the specifics of the controlling protocols including the awake time, the sleep mode, as well as the protocol units of data to proceed with the process of decision making. During the time when this method was proposed, the IEEE 802.21 had not been established as yet. Thus, they measured with this type of a flexible tool when managing the heterogeneous networks.

To date, studies have revealed various methods that emphasize the process of decision making by depending on the fuzzy logic [22], [23], or the techniques of multi-attributes 
decision-making [24]- [28], while accounting for certain aspects such as the mobility, data rates, RSS, speed, geolocation, and the distance among the APs. In [27], handover parameters optimization method is proposed based on ant colony algorithm. Simulations show that the proposed scheme outperforms the fixed parameters strategy.

The proposed study [29] hybrids a non-homogenous biogeography-based optimization (NHBBO) with a parallel fuzzy system (PFS). The PFSs are utilized to discover the probability of RAT selection, which acts as an input to the NHBBO procedure. PachecoParamo et al. [30] offered a VHO approach, which presented joint structures for admission control and access technology selection with vertical handoffs improve their capacity of radio resources in heterogeneous networks. Carvalho et al. [31] proposed optimal joint-call admission control (JCAC) for RAT selection in co-located wireless networks that can be apply on both non-real-time services and real-time services. El Helou et al. [32] suggested a hybrid method for RAT selection in heterogeneous wireless networks. They considered on access technology selection and formulate hybrid decision framework to combine user preferences and operator objectives dynamically [33]. However, these types of researches only concentrated on the process of decision-making and did not consider the standard IEEE 802.21 to carry out the decision-making as well as the supportive procedures including the collection and update of information, the VHO framework, and the management of data flowing among the interfaces of the networks.

To boost information distribution for IP-based vehicles, many developing mobile IP protocols can be utilized, which are completely under Internet Engineering Task Force (IETF). Also, IETF has developed MIPv6 to keep networking mobility (NEMO), named as the NEMO basic support protocol [34], where mobile network nodes (MNNs) can only be accessed through mobile router (MR). Obviously, there still are many problems for Mobile IP and NEMO, especially in highly dynamic traffic situations, such as end-toend transmission delay due to tunneling burden between home agent (HA) and MR, proper location for the HA, etc. To address these problems, some techniques for route optimization have been suggested [34]. Chen et al. [35] offered a new NEMO management structure where some neighboring vehicles with similar moving pattern are regarded as a virtual bus and all mobile routers can join to each other. In this way, the 
front mobile router can make the pre-handover process to reduce the handoff delay of the last mobile router.

\section{Network model}

This study's approach on the selection of the best network could be enhanced by utilizing the IEEE 802.21 standard of Media Independent Handover (MIH). This approach requires information regarding the access networks about the $\mathrm{MN}$ in order to make the right decision. The MIH standard is used to acquire several of the algorithm's decision inputs. This protocol helps the progression of the signaling message interchange between the handover decision unit and the different technologies for access. Thus, the MIH benefits from getting the significant information regarding the network and its users. Services are gained without any interruption by utilizing the qualifications and this standard's features with service qualities that meet the requirements of the users.

Various settings that establish the handover signaling in an integrated network such as the WiMAX, WiFi, as well as the UMTS are demonstrated in this study. The first signaling setting demonstrates the situation where a $\mathrm{MN}$ is located in an area that is overlapped and is able to choose a better connection by utilizing the $\mathrm{ABC}$ concept. Figure 1 depicts the MN using the overlapped areas of WiFi and WiMAX. The second setting demonstrates the signaling situation where a user is required to utilize the handover since the present connection would be lost due to the movement from a WiMAX network to the UMTS. These scenarios explain the way in which the MIH framework is able to provide a continuous service to a user including the approaches used to achieve the procedures.

The Point of Attachment as well as the Point of Service presented in the following is described based on the MIH outline. The network using MIHF that communicates directly with the MN's MIHF acts as the Point of Service of the specific mobile network. The information from the MIH is exchanged by the MN with the MIH's Point of Service via the L3 conduit if the Point of Service is present in the similar network to the Point of Attachment network. Point of Attachment is the network portion of a layer 2 link including the $\mathrm{MN}$ as the other end point. Thus, the MIH outline is supportive of the movements from the L2 as well as L3 in the exchange of information in the MIH. Two 
issues are considered during the decision-making process of a handover. First, the MN should aim to utilize a high bandwidth with an access network that is of low cost. Second, the count of irrelevant handovers should be lowered to prevent the degradation of the QoS of the present communication as well as to prevent network overload from the signaling traffic.

Several vertical handovers are involved in each mobile connection during the process of connecting. The mobile terminal is projected to get information from the collocating networks within the receiving frequency range. The information that is advertised in each network has the available bandwidth and the average delay, which is measured with the performance process IETF IP metrics. During every time interval, the MN establishes if the link should utilize the current network that is selected or redirected to other networks with better performance levels with lower costs, and higher guarantees of service quality. Redirection of the connection from one network to another includes a complex process that enhances the processing and signaling load of the network. Thus, the exchange takes place between the connection's QoS, the process as well as the signaling load [27].

\section{Formulate VHO decision making as optimization problem}

A significant challenge is the optimization of the process of vertical handover since a weak performance of optimizing could cause a drop or loss in the network signaling and power loss in the mobile device while advancing the QoS of the network. This study has designed an adaptive heuristic model aimed at achieving an optimized network during the decision-making stage of the vertical handover as well as a mobiles terminal that randomly moves along the heterogeneous wireless networks. The QoS parameter values are used to identify each network. The optimization issue deals with the attempt of aligning the weights of the QoS to determine the optimal network out of the available networks. The study demonstrates the benefits of the heuristic model in reaching an optimal solution that improves the performance offered by previous similar methods and algorithms [28].

An effective adjusting feature of the weights of the QoS that establishes beneficial network out of the available ones in the wireless network setting is essential. The benefits of each network that is available must be known to determine the best network. 
A metric or function must be designed to achieve this capability, which is able to acquire the benefits of the network. Firstly, each QoS parameter is assigned a set of weights to calculate the network's quality, which is based on the features of the network and the preferences of the user. An overall profile of the QoS parameter could be assigned a weight that ranges 0 to 1 . A specific function is responsible for this measure called the cost function. The evaluation of this function takes place in the phase of the $\mathrm{VH}$ decision-making. Thus, the optimization issue includes looking for the most beneficial solution with the lowest cost when applied to the networks and this would be chosen as the best solution for the VH decision-making phase.

The component of the $\mathrm{BBO}[36]$ allocates a relevant weight $\left(w_{1}, w_{2}, \ldots, w_{i}\right)$ for every initial decision based on the function objective identified by the operator in terms of importance and sensitivity to the selection criteria of the access network to various features of the wireless heterogeneous setting [37]. If $S=\left\{s_{1}, s_{2}, s_{3}, \ldots, s_{N}\right\}$ is considered as a set of candidate networks and $\mathrm{Q}=\left\{q_{1}, q_{2}, q_{3}, \ldots, q_{N}\right\}$ as a set of quality of service factors where $\mathrm{M}$ is the number of quality of service factors and $\mathrm{N}$ is the number of candidate networks. Additionally, each factor of QoS is considered to have its own weight that demonstrates the effect of the factor on the network or user. Consequently, We calculate cost function for each network based on Eq. (1) where $W_{N}$ is calculated using the analytic hierarchy process (AHP) [38]. This process is selected because of its ability to change its weighting between each factor based on network conditions and user preferences.

$$
C_{N}=W_{\text {Interface }} \times \sum_{j=1}^{M} q_{j} \times W_{j}
$$

With the above definitions, the AHP method can be described as follows: The relative scores among the QoS scores set are calculated and then Relative scores between any two scores are calculated using Eq. (2) where $R_{q_{i} q_{j}}$ is the relative score between parameters $q_{i}$ and $q_{j}$, and $S_{q_{i}}$ and $S_{q_{j}}$ are their respective scores. 


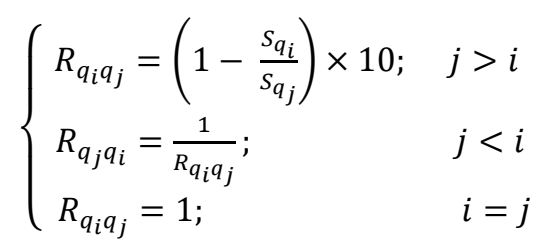

$X=\left\{X_{i j}\right\}$ is $M \times M$ matrix which $X_{i j}$ represents the priority scores of each factor is initialized as follows Eq. (3):

$$
X=\left[\begin{array}{ccccccc}
1 & R_{q 1 q 2} & R_{q 1 q 3} & R_{q 1 q 4} & R_{q 1 q 5} & \ldots & R_{q 1 q N} \\
\frac{1}{R_{q 1 q 2}} & 1 & R_{q 2 q 3} & R_{q 2 q 4} & R_{q 2 q 5} & \ldots & R_{q 2 q N} \\
\frac{1}{R_{q 1 q 3}} & \frac{1}{R_{q 2 q 3}} & 1 & R_{q 3 q 4} & R_{q 3 q 5} & \ldots & R_{q 3 q N} \\
\frac{1}{R_{q 1 q 4}} & \frac{1}{R_{q 2 q 4}} & \frac{1}{R_{q 3 q 4}} & 1 & R_{q 4 q 5} & \ldots & R_{q 4 q N} \\
\frac{1}{R_{q 1 q 5}} & \frac{1}{R_{q 2 q 5}} & \frac{1}{R_{q 3 q 5}} & \frac{1}{R_{q 4 q 5}} & 1 & \ldots & R_{q 5 q N} \\
\vdots & \vdots & \vdots & \vdots & \vdots & \ddots & \vdots \\
\frac{1}{R_{q 1 q N}} & \frac{1}{R_{q 2 q N}} & \frac{1}{R_{q 3 q N}} & \frac{1}{R_{q 4 q N}} & \frac{1}{R_{q 5 q N}} & \ldots & 1
\end{array}\right]
$$

Then when each element of the matrix $X$ is divided by the sum of its column Eq. (4), the normalized relative weight is obtained.

$$
X_{i j}=\frac{X_{i j}}{\sum_{i=1}^{M} X_{i j}}
$$

The normalized matrix $X$ is called $w_{\text {norm }}$ which is shown in Eq. (5).

$$
w_{\text {norm }}=\left[\begin{array}{ccccccc}
w_{11} & w_{12} & w_{13} & w_{14} & w_{15} & \ldots & w_{1 N} \\
w_{21} & w_{22} & w_{23} & w_{24} & w_{25} & \ldots & w_{2 N} \\
w_{31} & w_{32} & w_{33} & w_{34} & w_{35} & \ldots & w_{3 N} \\
w_{41} & w_{42} & w_{43} & w_{44} & w_{45} & \ldots & w_{4 N} \\
w_{51} & w_{52} & w_{53} & w_{54} & w_{55} & \ldots & w_{5 N} \\
\vdots & \vdots & \vdots & \vdots & \vdots & \ddots & \vdots \\
w_{N 1} & w_{N 2} & w_{N 3} & w_{N 4} & w_{N 5} & \ldots & w_{N N}
\end{array}\right]
$$

Next, the average values of each row are calculated to give the priorities for each factor by Eq. (6) which is shown in Eq. (7).

$$
\overline{w_{l}}=\frac{w_{i 1}+w_{i 2}+w_{i 3}+w_{i 4}+w_{i 5}+\cdots+w_{i N}}{n}
$$

The normalized vector Eq. (7) is called the priority vector. Since it is normalized, the sum of all the elements in priority vector is 1 . The priority vector shows relative weights 
among them.

$$
W_{N}=\left[\begin{array}{c}
\overline{w_{1}} \\
\overline{w_{2}} \\
\overline{w_{3}} \\
\overline{w_{4}} \\
\vdots w_{5} \\
\overline{w_{N}}
\end{array}\right]
$$

The AHP [38] is used to structure the problem and give the weights of selected criteria. There are studies in the literature that use the Technique for Order Preference by Similarity to Ideal Solution (TOPSIS) method [39] and grey relational analysis (GRA) [40]. But differently from other studies, M-BBO method is proposed for facility network selection and the results are compared in this study. Hwang and Yoon [39] was the first work that proposed TOPSIS. The proposed method was based upon the concept that selected alternative must have the farthest distance from negative ideal solution and the distance from the positive ideal solution should be the shortest. Positive ideal solution minimizes cost criteria and maximizes the benefit criteria while negative ideal solution minimizes the benefit criteria and maximizes the cost criteria and [39].

In continue, the module of the distance collision probability (DCP) is utilized to measure the border cell of the QoS, which ensures the QoS until a certain distance along the path. The module for the DCP calculates the conditions of the packet loss in order to achieve this; it is linked to the various networks at various distances from the vehicle and the Point of Attachment. AHP is used to calculate the initial weights and then DCP calculates maximum weights and then normalize weights to get the final weights.

This study has designed certain new algorithm proposals entrenched in intelligent computing which is able to overcome the issue of optimization in order to identify the best combination of quality of service parameters weights for a mobile terminal's heterogeneous wireless networks. Several parameters are assessed during the process of decision making to select the best candidate for a network. These parameters are derived from the processes carried out by the DCP module. The M-BBO takes into consideration the best suited $\mathrm{CN}$ to switch to as well as tries to choose the right timing to leave the prior Point of Attachment to attach itself to a new Point of Attachment. The estimation of model utilized here is selected based on the features of the applied underlying networks. 
A few models have been explained in the past literature [27-30]. In addition, models are measured by utilizing the geolocation and the status of the information network as calculated by the vehicles and stored in the database of the MIIS. According to the required $\mathrm{DCP}$, the $\mathrm{M}-\mathrm{BBO}$ ascertains if the $\mathrm{CN}$ can fulfill this type of requirements. Networks that have a lower DCP compared to the required minimum are not chosen.

Table 1 illustrates the probability of the collision [55] as the function of distance according to the interpolation of the curve fitting for the three networks' performance. The DCP's chosen threshold is $30 \%$. In relation to the weight value calibration $\omega$ i, this study has adopted the BBO process to measure each parameter's most suitable values for the various profiles of the users.

Table 1. Distance Collision Probability [55]

\begin{tabular}{|c|l|l|}
\hline DCP & Distance & \multicolumn{1}{c|}{ Collision probability } \\
\hline \multirow{4}{*}{ Wi-Fi } & $\mathrm{d} \leq 100$ & 1 \\
\cline { 2 - 3 } & $100<\mathrm{d} \leq 210$ & $0.5+0.01 . \mathrm{d}-0.001 . \mathrm{d}^{2}+2.8 \mathrm{e}^{-07} \cdot \mathrm{d}^{3}$ \\
\cline { 2 - 3 } & $210<\mathrm{d} \leq 250$ & $165.4-2.03 . \mathrm{d}+0.00833 \cdot \mathrm{d}^{2}-1.159 \mathrm{e}-05 \cdot \mathrm{d}^{3}$ \\
\cline { 2 - 3 } & $\mathrm{d}>250$ & 0 \\
\hline \multirow{4}{*}{ WiMAX } & $\mathrm{d} \leq 150$ & 1 \\
\cline { 2 - 3 } & $150<\mathrm{d} \leq 375$ & $0.4+0.007 . \mathrm{d}-3.485 \mathrm{e}^{-05} \cdot \mathrm{d}^{2}+4.258 \mathrm{e}^{-08} \cdot \mathrm{d}^{3}$ \\
\cline { 2 - 3 } & $375<\mathrm{d} \leq 500$ & $-44.908+0.333 \cdot \mathrm{d}-0.000798 \cdot \mathrm{d}^{2}+6.2 \mathrm{e}^{-05} \cdot \mathrm{d}^{3}$ \\
\cline { 2 - 3 } & $\mathrm{d}>500$ & 0 \\
\hline \multirow{3}{*}{ UMTS } & $\mathrm{d} \leq 200$ & 1 \\
\cline { 2 - 3 } & $200<\mathrm{d} \leq 310$ & $0.62+0.005 . \mathrm{d}-5.95 \mathrm{e}-05 . \mathrm{d}^{2}+4.258 \mathrm{e}-08 . \mathrm{d}^{3}$ \\
\cline { 2 - 3 } & $310<\mathrm{d} \leq 550$ & $-10.908+0.8 \cdot \mathrm{d}-0.09 \cdot \mathrm{d}^{2}+9.2 \mathrm{e}^{-05} \cdot \mathrm{d}^{3}$ \\
\cline { 2 - 3 } & $\mathrm{d}>550$ & 0 \\
\hline
\end{tabular}

\section{Hybrid Markov chain and Biogeography-based optimization}

The subsequent sections will describe the methods used to design the decision problem of the vertical handoff as the process of a Markov chain [41]. The vehicular establishes the course of action when it has passed the time duration. As the vehicular velocity has physical property constraints and speed in the future is not influenced by the past one, this study has adopted the Markov chain model suggested by [41] to define the mobility model. Shadow fading as well as the mobility of the vehicular might result in the signal attenuation in the wireless environment. 
A process that is random with a distinctive group of potential state values $s_{i}(\mathrm{i}=1, \ldots$, $\mathrm{T}$ ) is known as the Markov chain. The state of the system at time $t$ can be described by a pair of random variables, $s(t)_{i}$ and $s(t)_{j}$, specifying the number of calls present at a time $\mathrm{t}$ for the serving network and the selected network during vertical handover process. The system's transition probability from state $s_{i}$ to $s_{j}$ is defined by the probability $p_{i j}$ or the probability of transition. The $\mathrm{T} \times \mathrm{T}$ matrix $\mathrm{P}=\left[p_{i j}\right]$ is known as the matrix of transition. The chain is considered regular if it can shift from any state to another and it need not be in a single step. The normal Markov chains' fundamental limitation theorem claims that when $\mathrm{P}$ is normal, the following equation Eq.(8) is possible:

$$
\lim _{n \rightarrow \infty} P^{n}=P_{s s}
$$

Whereby every row $p_{s s}$ of $P_{s s}$ is similar. The ith component of $p_{s s}$ represents the probability of the Markov chain in state $s_{i}$ using transitions that are infinitive. $p_{s s}$ is not dependent on the initial state. A Markov state in the BBO represents a BBO distribution of population. The probability $p_{i j}$ is the probability of the transitions of the population from the $s_{i}$ distribution to the $s_{j}$ distribution following a single generation. Should the rate of mutation be non-zero, the probability is considered higher than zero, denoting a regular transition matrix. It means a distinctive non-zero limiting probability is present for every potential population distribution as the number of generation reaches infinity. If the BBO is not inclusive of mutations, then it is possible to converge into a uniformed population, as in a population with identical individuals; this form of Markov chain is also known as being absorbing. The probability of the convergence of the population can be measured in every state as well as the projected convergence time. $\mathrm{P}(\mathrm{v})$ represents the $\mathrm{N} \times \mathrm{n}$ matrix that includes the probability of getting each $\mathrm{n}$ possible individuals at every $\mathrm{N}$ trial, and here only the migration is taken into consideration. $P^{(2)}(\mathrm{v})$ includes the probability of the migration as well as the mutation. In this scenario, the probability of transitioning from population vector $\mathrm{v}$ to $\mathrm{u}$ after one generation is symbolized by the following equation Eq.(9):

$$
\operatorname{Pr}^{(2)}(u \mid v)=\prod_{k=1}^{N} \prod_{i=1}^{n}\left[P_{k i}^{2}(v)\right]^{J_{k i}}
$$


where Eq. (9) is utilized to look for the matrix of transition for the BBO with the mutation and migration. Biogeography refers to the study of geographical distribution of species over geological time frames. There is extensive literature on biological subjects. In 2008, Simon [36] first utilized the biogeography analogy to the concept of engineering optimization and introduced the BBO approach. This is a population-based method that works with a set of candidate solutions across generations. It examines the combined big solution spaces using a stochastic method as used by most other evolutionary algorithms [36]. It copies the species' geographic distribution to present the problem and the solution candidates in the search location, utilizing the specific mutation and migration process to re-distribute the solution instances over the search location in search of the solutions that are almost optimal globally. BBO is different because BBO has been examined in different combinations and constrained/unconstrained optimization challenges [42] involving such as the Traveling Salesman Problem [43], [44], classification of satellite image [45], as well as sensor selection [46] among others.

Nevertheless, since 2012, research using BBO as a technique for choosing genes for data analysis of microarray gene expression has not been reported. There is an ecosystem or population in the BBO that possesses some of the island habitats. Every habitat contains the index of habitat suitability that is the same as the fitness function which relies on most of the island's traits or attributes. When a value is given to every trait, then habitat H's HSI is these values' function. These variables that collectively characterize the suitability of the habitat formulate the suitability index variables (SIVs).

In terms of the issues related to the selection of genes, a habitat's SIVs (solution candidate) are the chosen subset of the genes derived from the grouping of the entire genes. Therefore, the ecosystem is a randomized group of gene candidate subsets. A proper solution is analogous to a proper HSI and vice versa. Proper solutions of HSI are likely to share the SIVs with weak solutions of HSI. This type of sharing, which is known as migration, is governed by the habitats' rates of immigration and emigration. The model has been purposefully maintained uncomplicated and followed the original simple linear migration model as demonstrated in Figure 2. 
Whereby $\mathrm{E}$ and I represent the maximum rates of emigration as well as immigration, which are normally fixed at 1 . Individual rates of immigration as well as emigration $(\lambda$ and $\mu$, accordingly) are measured using a similar formula as the simple linear model suggested by [36].

This section covers the proposed algorithm for the M-BBO according to the algorithm of the $\mathrm{BBO}$. $\mathrm{BBO}[36]$ contains two main stages namely migration as well as mutation. A mechanism for mutation in the proposed $\mathrm{M}-\mathrm{BBO}$ is engaged in improving the capability of investigating in the search location. The detailed algorithm for the BBO can be retrieved from [36]. The subsequent sub-sections report the proposed algorithm of the MD-PBBO for optimization of the weight coefficients for choosing the best RAT in the networks that are heterogeneous.

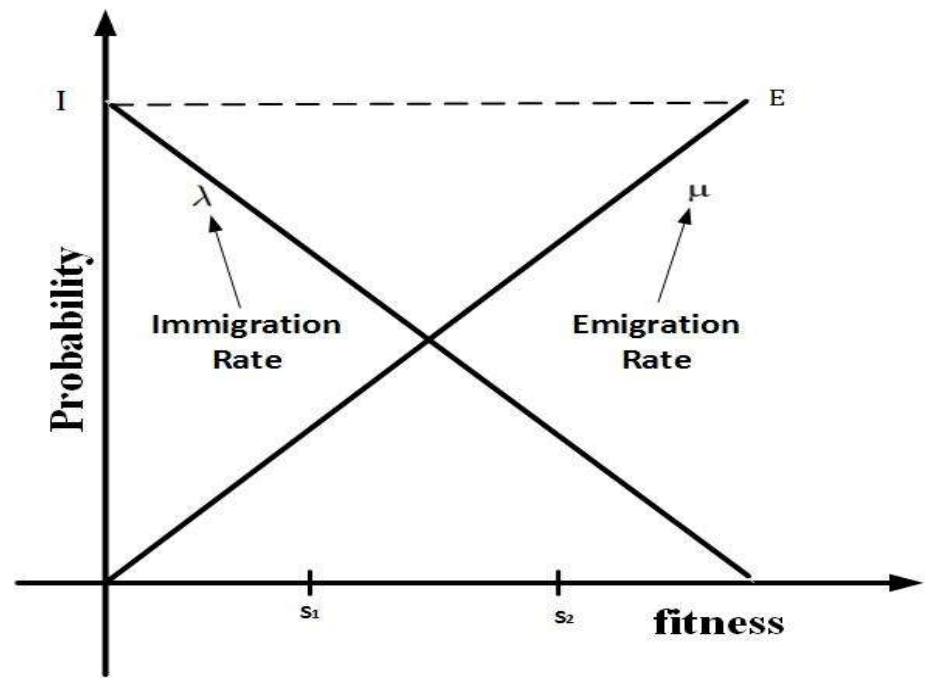

Figure. 2. Rate of Migration versus Number of Species [36]

In general, studies normally apply different ideas to generate a feasible solution by managing the quantity of diversity. The process of mutation in the BBO improves the population diversity. It should be realized that the rate of the mutation changes the SIV of the habitat in a randomized approach according to the rate of mutation. In addition, the rate of mutation is inverse in proportion to the species count probability. Therefore, in a fundamental $\mathrm{BBO}$, if a solution is chosen for mutation, it will be replaces using a random method to develop a new set of solution. Thus, this randomized mutation influences the investigation of the basic BBO capability. The process of mutation is modified to enhance the investigating ability of the BBO to refine the habitat and to 
reach an optimal solution using a better method. For the BBO algorithm, a short introduction is provided; then, then, a pseudo code is used to explain the operation.

The species selection (Ps) probability changes from a specific time to another as shown in Eq. [9] in this paper. Changes are not performed in the migration portion of the proposed $\mathrm{M}-\mathrm{BBO}$ algorithm, to sustain the ability to exploit. The modification performed in the mutation section with the $\mathrm{M}$ improved the capability for investigation. Therefore, the proposed M-BBO leads to a balanced investigation and the ability to exploit the algorithm. The proposed M-BBO algorithm's pseudocode is presented in Figure 3.

\section{Pseudo code for proposed M-BBO algorithm.}
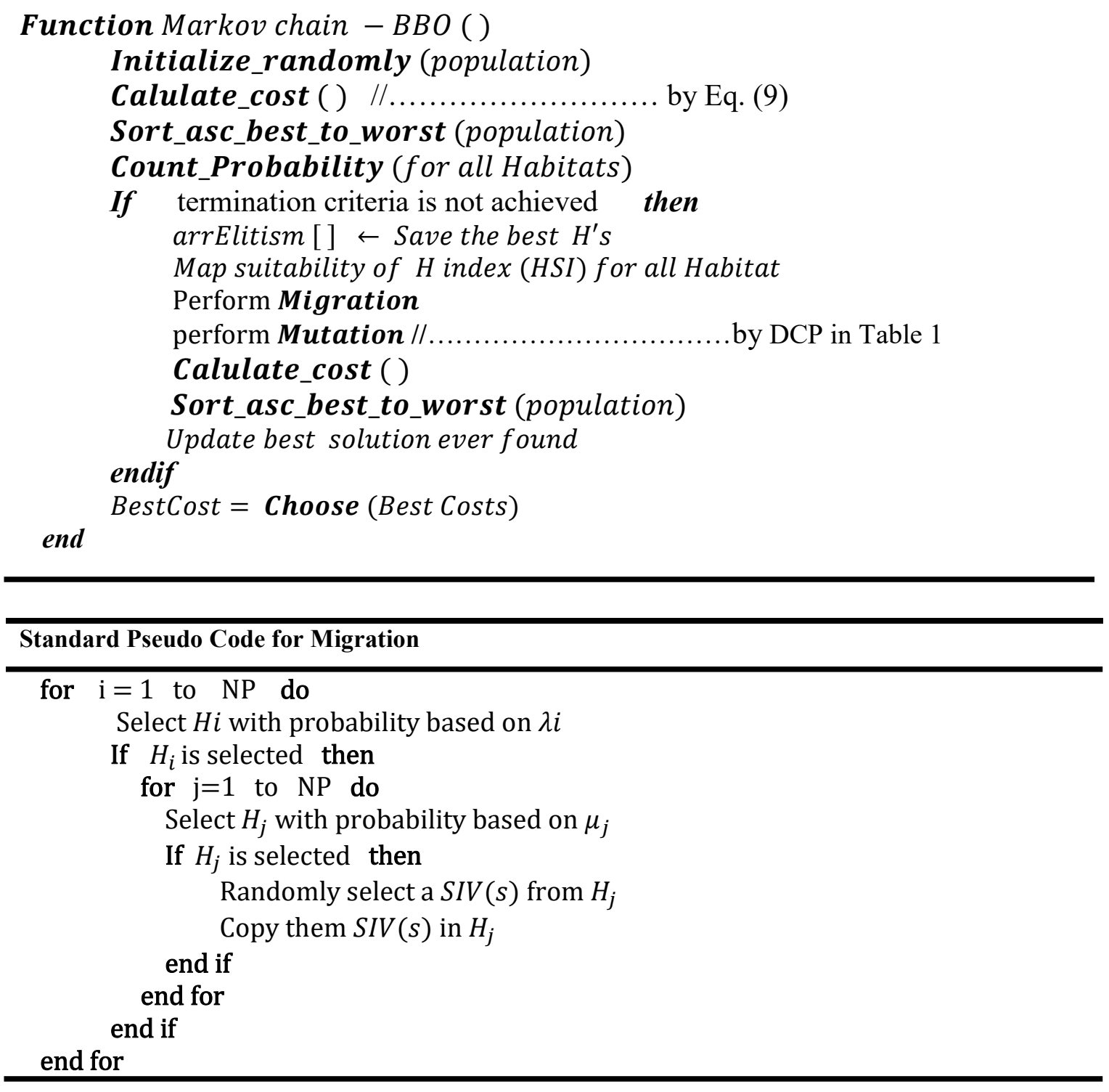


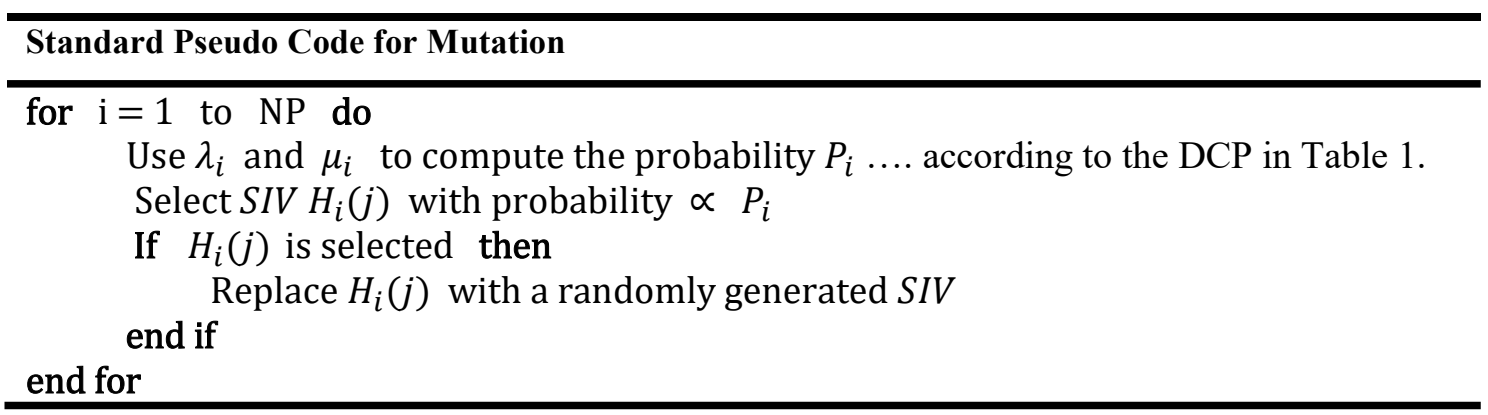

Figure 3. Pseudo code for proposed M-BBO algorithm

The proposed M-BBO algorithm is used in this study to perform the optimization of weight in an algorithm with a multi-point decision making and to choose the best RAT for the considered networks that are heterogeneous

Let us consider having a group of candidate solutions for specific challenges. Specific characteristics are used to identify each candidate solution. The probability of the shared characteristics of the solutions according to the fitness of the value solutions is represented by the $\mathrm{BBO}$. The $\mathrm{s}$ feature is said to have emigrated from the $\mathrm{x}$ solution and immigrated to the y solution in the BBO when a copy of the s replaces a feature in the $\mathrm{y}$. The probability of the x-solution sharing its characteristics with other individuals in the population is in proportion to the $\mathrm{x}$ fitness. The probability of the $\mathrm{y}$ solution accepting a feature from the individuals in the population reduces with the fitness of $y$. These probabilities of migration depend on the curves, as demonstrated in Figure 2. To simplify, it is assumed that all the solutions have similar migration curves. Figure 2 denotes two solutions in the BBO. S1 denotes a poor solution, while S2 denotes a betterfit solution. The probability of immigration for S1 will, in turn, be higher compared to the probability of immigration for S2. The probability of emigration for S1 would be lesser compared to the probability of emigration for S2.

For every feature in each solution in this approach, it is probabilistically determined if immigration should be carried out. If a particular feature immigrates, then the solution that is emigrating is chosen based on the probability of the fitness by utilizing the roulette wheel selection. Figure 3 demonstrates this algorithm as the explanation of one generation of the BBO. Migration and mutation of the whole population occurs prior to replacing any of the solutions in the population that needs the utilization of the 
temporary population $\mathrm{z}$ in the algorithm. The entire modeling process is shown in Figure 4.

\section{Performance Evaluation}

The computational results are obtained by developing M-BBO and BBO in MATLAB. Also, to implement the framework, OMNeT++ has been utilized as an efficient, flexible and a discrete network simulator (OMNeT++ User Manual). Table 2 shows the performance of the proposed optimization search strategies, $\mathrm{M}-\mathrm{BBO}$ and $\mathrm{BBO}$, using simulations.

Standard BBO and M-BBO have been compared to each other based on a set of realworld benchmarks to demonstrate the improvement of performance. Regarding BBO, linear migration curves [36], maximum immigration and emigration rates of 1 and a mutation probability of 0 have been used. A population size of 50 has been used for each algorithm with a fitness function evaluation limit of 100000 and elitism size of 2 . The difference between $\mathrm{M}-\mathrm{BB}$ ) and standard BBO can be considered as using fitness-based selection by standard $\mathrm{BBO}$ while probability distribution of the population sizes for selection is used by M-BBO. The data in Table 2 for standard BBO is taken from [52]. The computational time for both standard $\mathrm{BBO}$ and $\mathrm{M}-\mathrm{BBO}$ is the same because the algorithms execute identically; however, in fitness based selection and probability-based selection, it would be different.

Wilcoxon method has been used to test for statistical significance [53]. Table 2 presents the Wilcoxon test results. According to the table, if the difference between the pair of algorithms is statistically noteworthy, the pair is marked. In order to to compare BBO with M-BBO, real-world optimization problems from the 2011 IEEE Congress on Evolutionary Computation (CEC) [54] has been used. We used some functions such as Ackley function, unimodal one-max problem, multimodal problem and deceptive problem with 20 dimensions to confirm the difference between $\mathrm{M}-\mathrm{BBO}$ and $\mathrm{BBO}$. The results in Table 2 are divided the BBO versus $\mathrm{M}-\mathrm{BBO}$ group. For each pair of algorithms, B/S/W scores have been calculated, where "W" denotes the number of times that the left algorithm performs worse than the right one, " $\mathrm{B}$ " shows the number of times 
that the right algorithm performs better than the left one and " $\mathrm{S}$ " presents the number of times that the left algorithm performs statistically the same as the right, and.

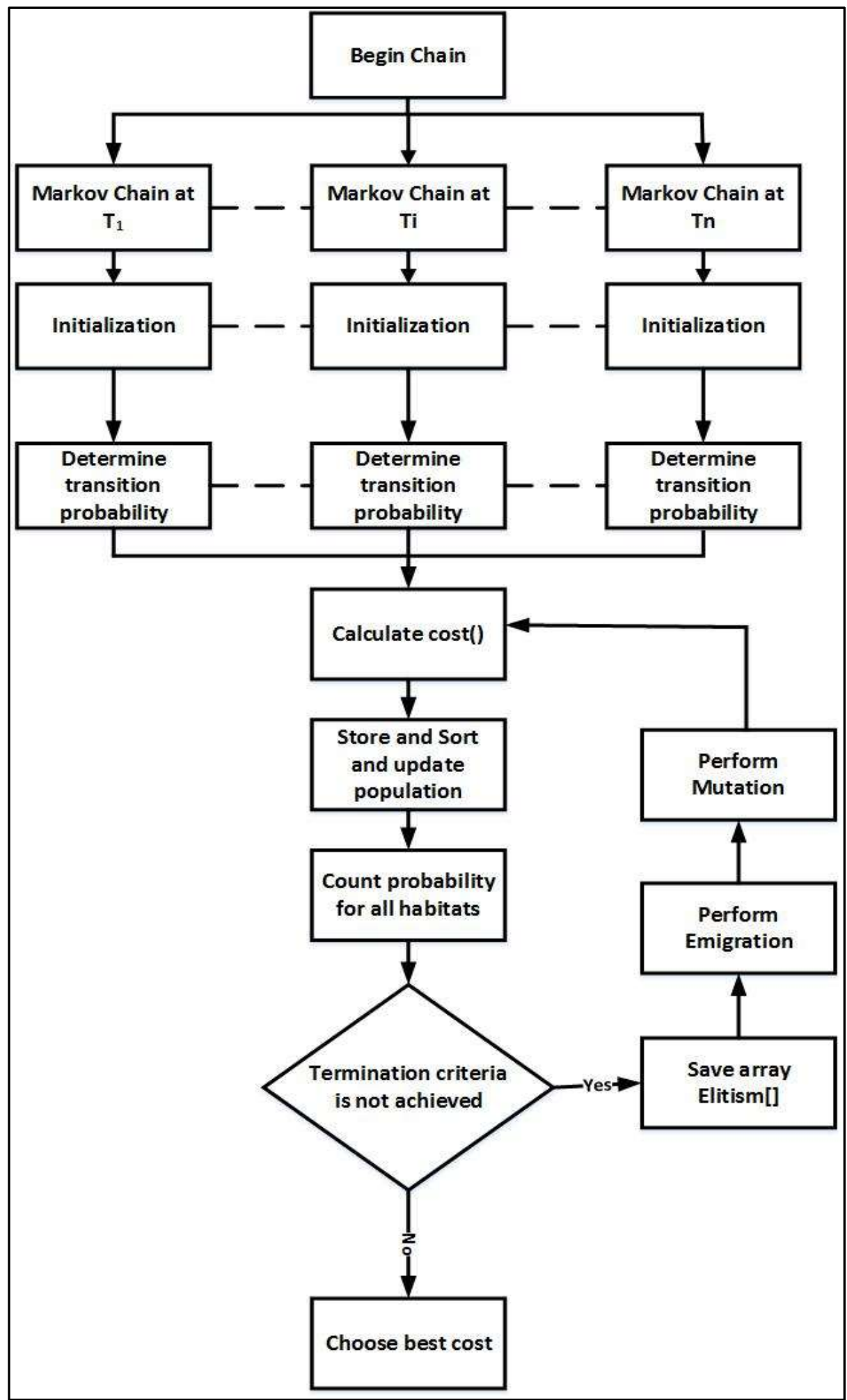

Figure 4. Flow chart of the M-BBO model 
The pairs are marked as follows when the difference between the algorithms is statically considerable, "O-X" shows that the right algorithm is better than the left one, "X-O" demonstrates that the left algorithm is better than the right one, the $\mathrm{B} / \mathrm{S} / \mathrm{W}$ row at the bottom shows the total scores. Comparing BBO versus $\mathrm{M}-\mathrm{BBO}$, Table 2 shows that, the $\mathrm{B} / \mathrm{S} / \mathrm{W}$ score is $1 / 2 / 8$, which indicates that $\mathrm{BBO}$ outperforms $\mathrm{M}-\mathrm{BBO}$ one time, $\mathrm{BBO}$ is statistically the same as M-BBO two times, and M-BBO outperforms BBO 8 times.

In addition, the OMNET++ is used to evaluate the performance of the network. The MBBO parameter algorithm is demonstrated in Tables 3 and 4. Average time is set at $15 \mathrm{~s}$ for the successive decision epochs [47]. $16 \mathrm{~kb} / \mathrm{s}$ is set as the bandwidth unit, while $2.5 \mathrm{~ms}$ is the jitter unit, and 0.5 erl is the traffic unit. 1 and 5 units are set as the highest and lowest velocities as used by [48], [49]. The size of the area of the cell is 3 times bigger compared to the WLAN while the size of the spatial density of the mobile network within the network of the cell is 8 times bigger compared to the WLAN. The WiMax DL's peaking data rates include $75 \mathrm{Mbps}$ UL: $25 \mathrm{Mbps}$, and DL: 100 to $324.6 \mathrm{Mbps}$ UL: 50 to $86.4 \mathrm{Mbps}$ in the UMTS. The VHO algorithm that is offered in this study is assessed with the Order of Preference Technique by Similarity to Ideal Solutions [36] based on the average handoffs amount, bandwidth that is available, and so on.

Table 2. Wilcoxon Test Results for Algorithms Comparisons.

\begin{tabular}{|l|c|}
\hline \multicolumn{1}{|c|}{ Function } & BBO vs M-BBO \\
\hline Ackley function & O-X \\
\hline Unimodal one-max problem & O-X \\
\hline Multimodal problem & O-X \\
\hline Deceptive problem & X-O \\
\hline Large Scale Transmission Pricing Problem & O-X \\
\hline Hydrothermal Scheduling Problem & O-X \\
\hline Circular Antenna Array Design Problem & O-X \\
\hline Transmission Network Expansion Planning (TNEP) Problem & O-X \\
\hline Bifunctional Catalyst Blend Optimal Control Problem & - \\
\hline Lennard-Jones Potential Problem & O-X \\
\hline Static Economic Load Dispatch (ELD) Problems & $1 / 2 / 8$ \\
\hline \multicolumn{1}{c}{ B/S/W } & \\
\hline
\end{tabular}


The MIIS provides information about the networks that are available and the respective PoAs encompassed in the simulated area. Table 2 denotes the key configuration determined for the experiments. One UMTS, five WiFi, and a single WiMAX Point of Attachment encompass the different location with different data rates. Moreover, each network in this scenario is configured based on various performance parameters. Various alternatives are designed for the assessment of the CNs using this approach.

This scheme is found to be a suitable compromise between the technology description accuracy and the high level of abstraction, which allows short simulations, for testing and comparing the efficacy of the various network selection algorithms. OMNeT++ has a structural component, denoted by the composed modules with hierarchical structures having any number of levels, and a behavioural feature, denoted by simple modules, as defined in $\mathrm{C}++$. Messages are utilized to realize the communication between modules. The OMNeT++ includes a tool for debugging and visualization, randomized number generators, statistics collection, etc.

All the simulations in Figure 5 were run at 100000 seconds simulation time while for every file length, there were 10 different runs, where these results of the values are the average of the 10 runs. The IP packets contain a length of 1000 bytes in simulations, so that it is compatible with $[50,51]$.

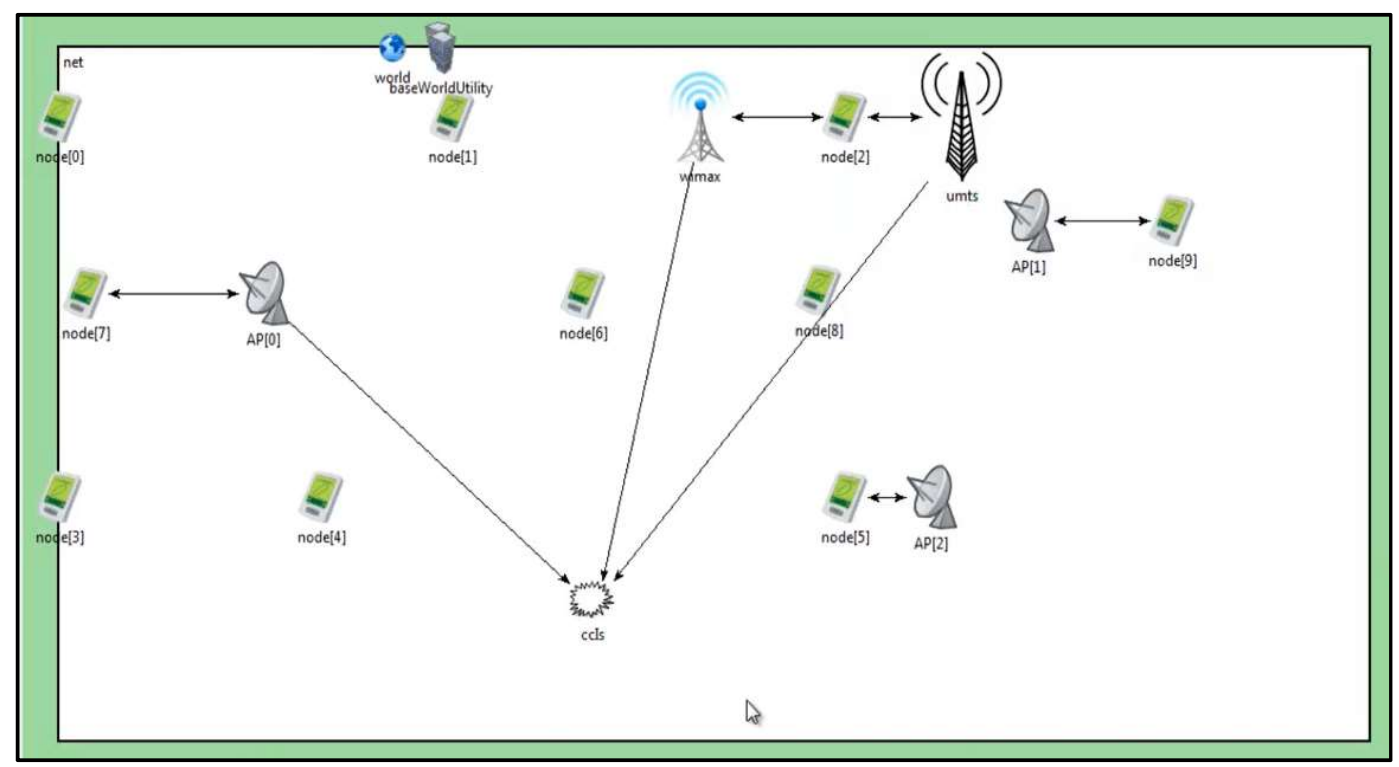

Figure 5. Simulated scenario in OMNET++ 
In addition, several tests were performed with various $\mathrm{MN}$ speeds. Initially in the simulation, the amount of the vehicular are not much however at the time of the simulation, the researchers try increasing the vehicular slowly to examine the functioning of the model that is proposed in an environment with high traffic. A GPS add-on module has also been implemented for the OMNET++ that handles the GPS coordinates, maps, and routes, to choose the itinerary to travel from the present geolocation to any other destination. The GPS module also interprets the coordinates of the geolocation into traveling time, thus allowing the algorithm of the M-BBO to recognize the place where the vehicle is anticipated to be at a given time in the future. Figure 5 shows the itinerary covering a distance of $6.50 \mathrm{~km}$ in a $4.50 \mathrm{~km}^{2}$ area. The GPS module manages the itinerary's entire coordinates. In addition, the MIIS informs about the networks and their respective Points of Attachment that are available in the simulated location as demonstrated in Figure 6. One UMTS, five WiFi, and one WiMAX PoA that cover various areas with distinctly offered data rates are observed. It is critical to note that the UMTS encompasses the whole setting, which means that the UMTS technology is always the backup connection technology for this group of trials.

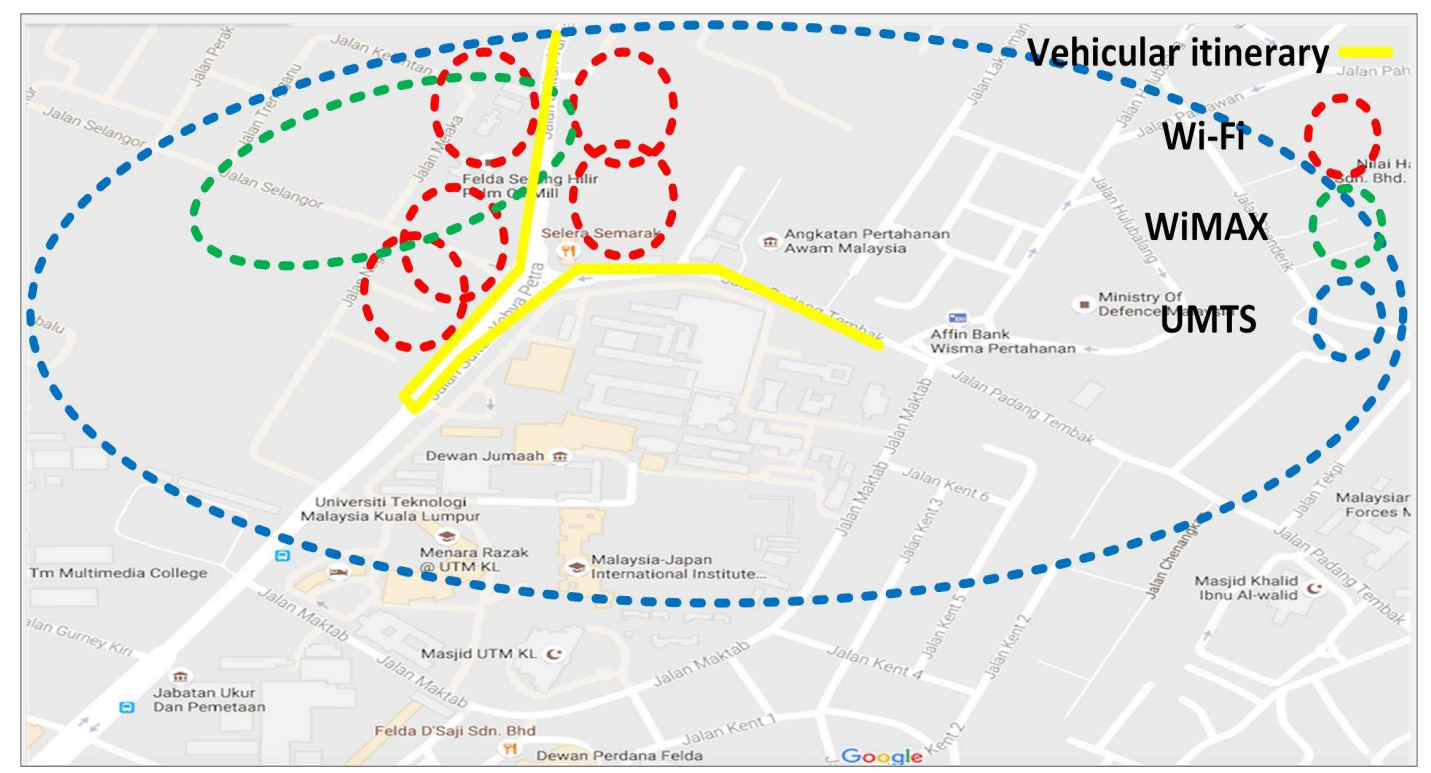

Figure 6. Coverage scenario

The parameters of the networks are shown in Table 4. The least requirement needed for the video session using the network that is chosen in the simulation is presented in Table 
5. Since the function of the video session is expected to be the main function in the increase of the future demands in mobile applications, the video streaming traffic has been emphasized.

Table 3. M-BBO Elements

\begin{tabular}{|c|c|c|c|}
\hline Elements & WiFi & WiMAX & UMTS \\
\hline Access Points & 5 & 1 & 1 \\
\hline Data rates (Mbps) & 28.5 & 17.3 & 3.5 \\
\hline VHO latency (ms) & 1080 & 2665 & - \\
\hline Coverage(m) & 500 & 1000 & 5000 \\
\hline Advertisement interval & 100 & 5000 & - \\
\hline
\end{tabular}

Average time is set at $15 \mathrm{~s}$ [43] for the continuous decision timing. $16 \mathrm{~kb} / \mathrm{s}$ is set as the bandwidth unit, while $2.5 \mathrm{~ms}$ is the jitter unit, and $0.5 \mathrm{erl}$ is the traffic unit. 1 and 5 units are set as the highest and lowest velocities as used by [43]. The size of the area of the cell is 3 times bigger compared to the WLAN while the size of the spatial density of the mobile network within the network of the cell is 8 times bigger compared to the WLAN. Figures 6-10 represent the network performance of the handoff setting.

Table 4. Summarized Network Parameters

\begin{tabular}{|c|c|c|c|c|c|}
\hline PoA & Technology & Price(MB) & $\begin{array}{c}\text { Latency } \\
\text { (Packet) }\end{array}$ & $\begin{array}{c}\text { Ratio of Packet } \\
\text { Loss }\end{array}$ & $\begin{array}{c}\text { Throughput } \\
\text { (Mbps) }\end{array}$ \\
\hline PoA-1 & WiFi & 0.8 & 15.44 & 1.19 & 1.48 \\
\hline PoA-2 & WiMAX & 0.15 & 17.59 & 2.74 & 1.18 \\
\hline PoA-3 & UMTS & 0.9 & 25.22 & 0.76 & 1.42 \\
\hline PoA-4 & WiFi & 0.8 & 15.40 & 1.09 & 1.40 \\
\hline PoA-5 & WiFi & 0.8 & 14.48 & 1.15 & 1.45 \\
\hline PoA-6 & WiFi & 0.8 & 14.34 & 1.10 & 1.38 \\
\hline PoA-7 & Wi-Fi & 0.8 & 25.40 & 1.29 & 1.43 \\
\hline
\end{tabular}

Table 5. Values of weights in the cost function for preferences of users

\begin{tabular}{|l|c|c|c|c|}
\hline \multicolumn{1}{|c|}{ AP } & Cost & Streaming & Conversational & $\begin{array}{c}\text { Maximum } \\
\text { performance }\end{array}$ \\
\hline Latency & 0.1057680 & 0.4345300 & 0.1657905 & 0.0623170 \\
\hline Packet Loss & 0.0224017 & 0.1432380 & 0.2545390 & 0.3898690 \\
\hline Throughput & 0.3733550 & 0.0234577 & 0.3435154 & 0.4845670 \\
\hline Price (MB) & 0.4534210 & 0.4176509 & 0.2467543 & 0.0367890 \\
\hline
\end{tabular}


Although various vertical handoff decision algorithms have been proposed in the literature recently, there is a lack of performance comparisons between classical TOPSIS and GRA methods and heuristic schemes. The number of handovers is recorded with the proposed scheme, (GRA) [40], as well as (TOPSIS) [39]. The rate of handoff using the GRA and the TOPSIS gets higher as more MNs join the network. The rate of handoff in the scheme that is proposed in comparison with the GRA and the TOPSIS is demonstrated in Figure 7(a). In this simulation, the total number of vehicular is fixed at 100 vehicular. Among the reasons seen during the simulation is the unsuitable handover that is triggered because of the RSS in relation to the GRA and TOPSIS. The technique for the proposed handover triggering lowers the rate of handoff significantly.

Likewise, the numbers of failed handovers are examined during the simulation. The number of failed handovers for GRA and TOPSIS is high due to the triggering method. The GRA and TOPSIS start frequent handovers that need quick switching of interfaces between various technologies. Hence, the MN consumes a lot of energy because of the interface switching. In this research, the energy usage of the $\mathrm{MN}$ is not taken into consideration. Figure 7 (b) demonstrates the comparison of the proposed scheme, GRA, and TOPSIS based on the failed handovers.

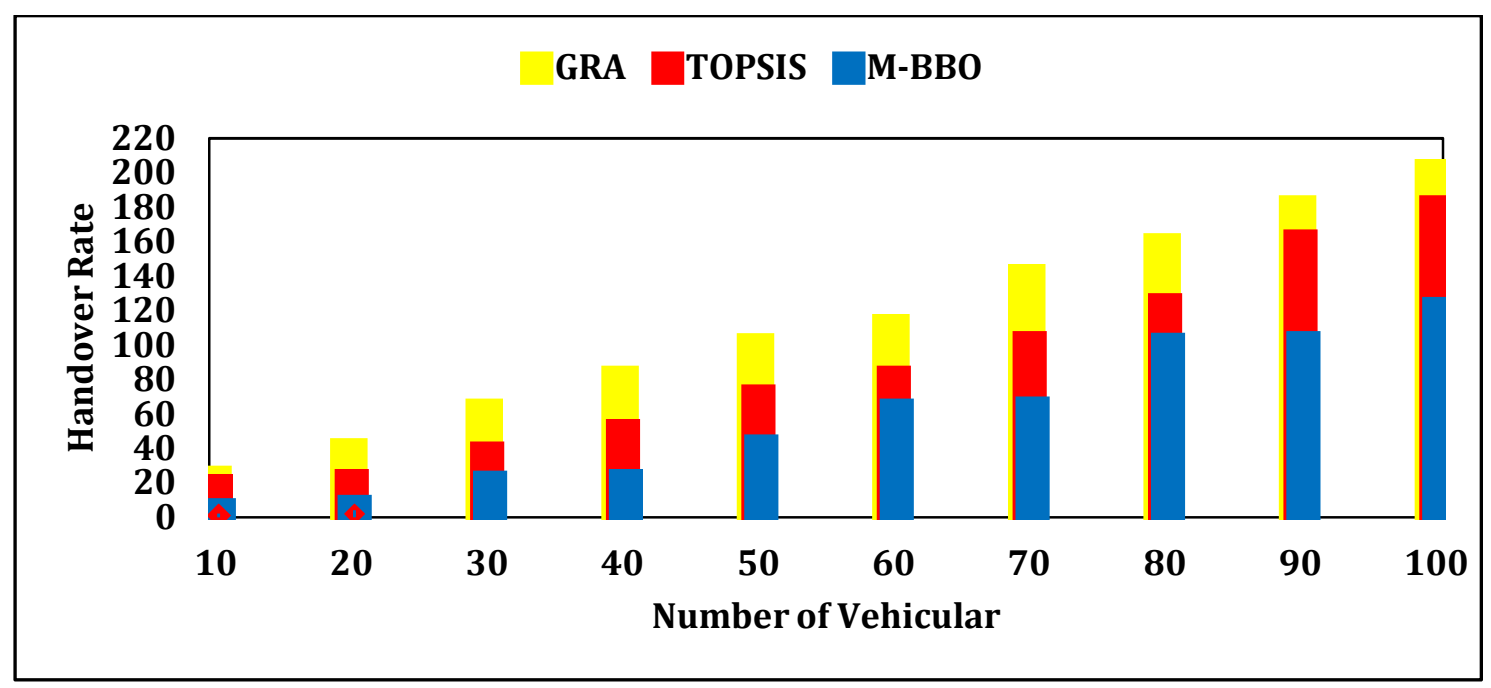

Figure 7 (a). Analysis of rate of handover

Similarly, the packet loss is significantly minimized in the proposed scheme. Each vehicle is moving over the range from $10 \mathrm{~km} / \mathrm{h}$ to $100 \mathrm{~km} / \mathrm{h}$. This simulation was performed with a packet size of 320 bytes and packet rate of 100 packets/sec. We have 
provided simulation results and compared the results with GRA, TOPSIS and NEMO [35]. The GRA and the TOPSIS have a high packet loss in comparison to the proposed scheme due to the regular switching of various networks. The packet loss rates of the GRA and TOPSIS schemes raises faster than that of the NEMO and M-BBO schemes due to the fact that higher vehicle speed can only tolerate low level of handover latency and the handover latency of the GRA and TOPSIS schemes are higher than that of the NEMO and M-BBO schemes and thus suffers higher packet loss rate. In general, a scheme with a multi-criteria decision needs a high level of handover time in comparison to a model with a single criteria decision. However, because of the proposed M-BBO method, the vehicular has additional time to scan as well as choose the optimized network in a network setting that is heterogeneous.

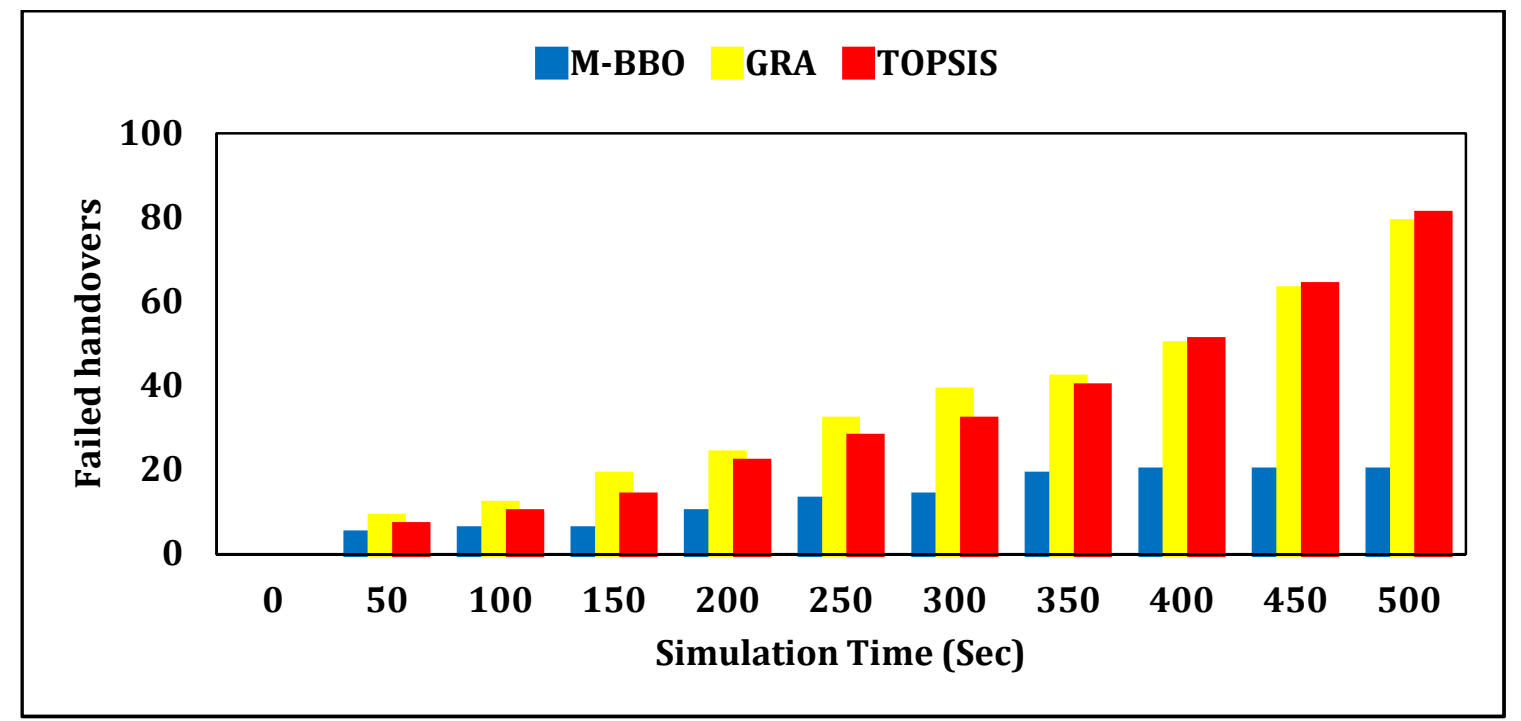

Figure 7 (b). Analysis of the failed handover attempts

Figure 7 (c) demonstrates the packet loss ratio comparison. As we can see, the packet loss rate of the M-BBO scheme is the lowest, followed by the NEMO, TOPSIS and GRA schemes. The scheme that is proposed has also enabled the computation of the throughput gain. The throughput relies on the loss of the packets indirectly. The GRA and TOPSIS possess high loss of packets and as such, they offer a low throughput gain due to unsuitability in the selection of the network for the handover. However, the proposed scheme also faces a lower packet loss due to the optimal network selection. The throughput relies on the delay of the handover and the needed time to redirect the data via a new network. The handover that is proposed offers the vehicular sufficient 
time while the handover occurs. Thus, the data is redirected via a network that is new, and as such, the vehicular goes through a high level of throughput.

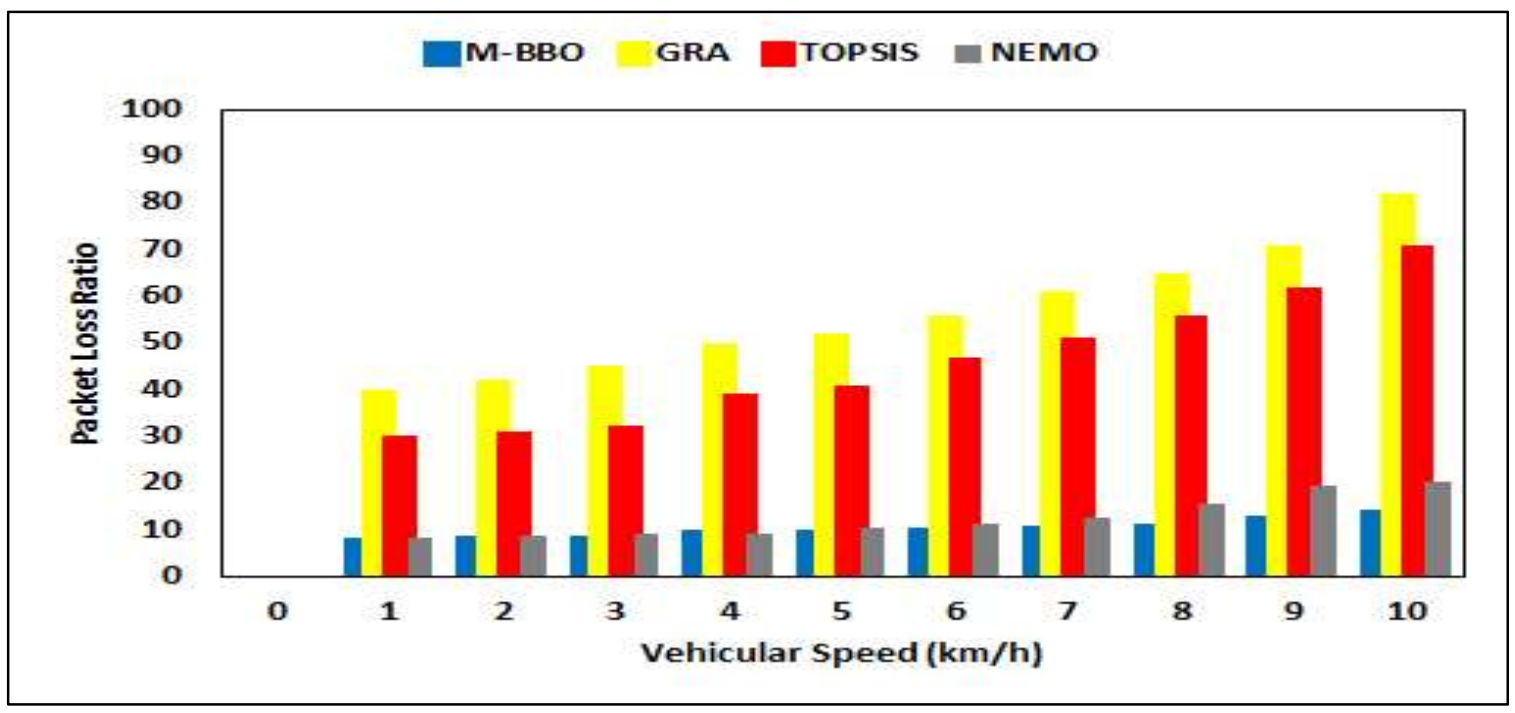

Figure 7 (c). Packet loss during handover.

Figure 7 (d) shows the throughput gain comparison in the proposed scheme, GRA, and TOPSIS decision models. At first, the vehicular has a low level of throughput, however, after certain duration, the throughput increases. Two reasons for this increase include i) the previous throughput (bytes) arriving through the present AP/BS is added to the new bytes arriving from the AP/BS that are new, and ii) the proposed trigger and network selection offer the vehicular with a suitable AP/BS that increases the throughput.

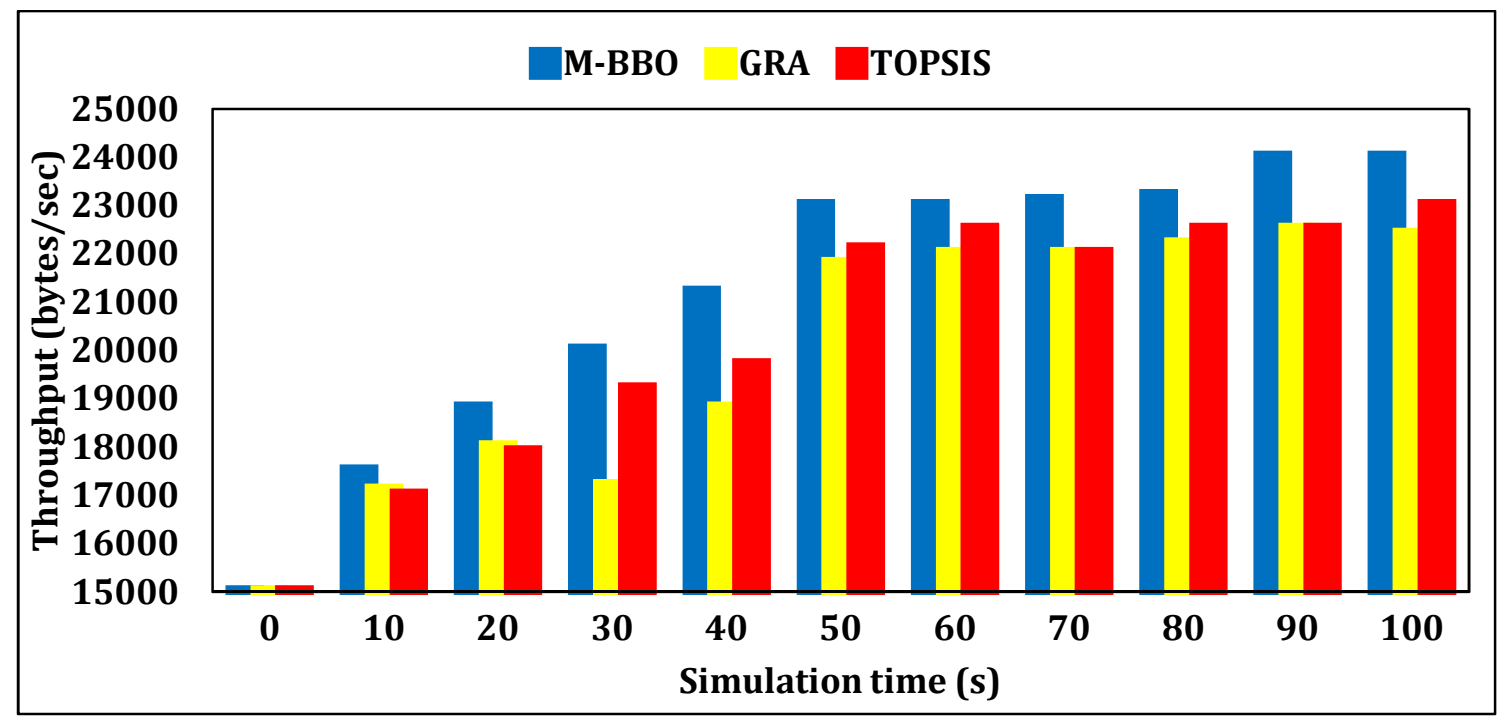

Figure 7 (d). Throughput analysis. 
Figure 7 (e) and Figure 7(f) present the handover latencies of the M-BBO, NEMO, TOPSIS and GRA. The handover latency of the M-BBO and NEMO methods are lower, followed by the TOPSIS and GRA methods. Figure 7 (e) shows the impact of vehicular speed to handover latency. In this simulation, the total number of vehicular is fixed at 50 vehicular. Whenever the vehicular speed rises, the handover latency also rises. The MBBO and NEMO models have better performance than the TOPSIS and GRA models because they have high level of handover time and thus increase the handover latency.

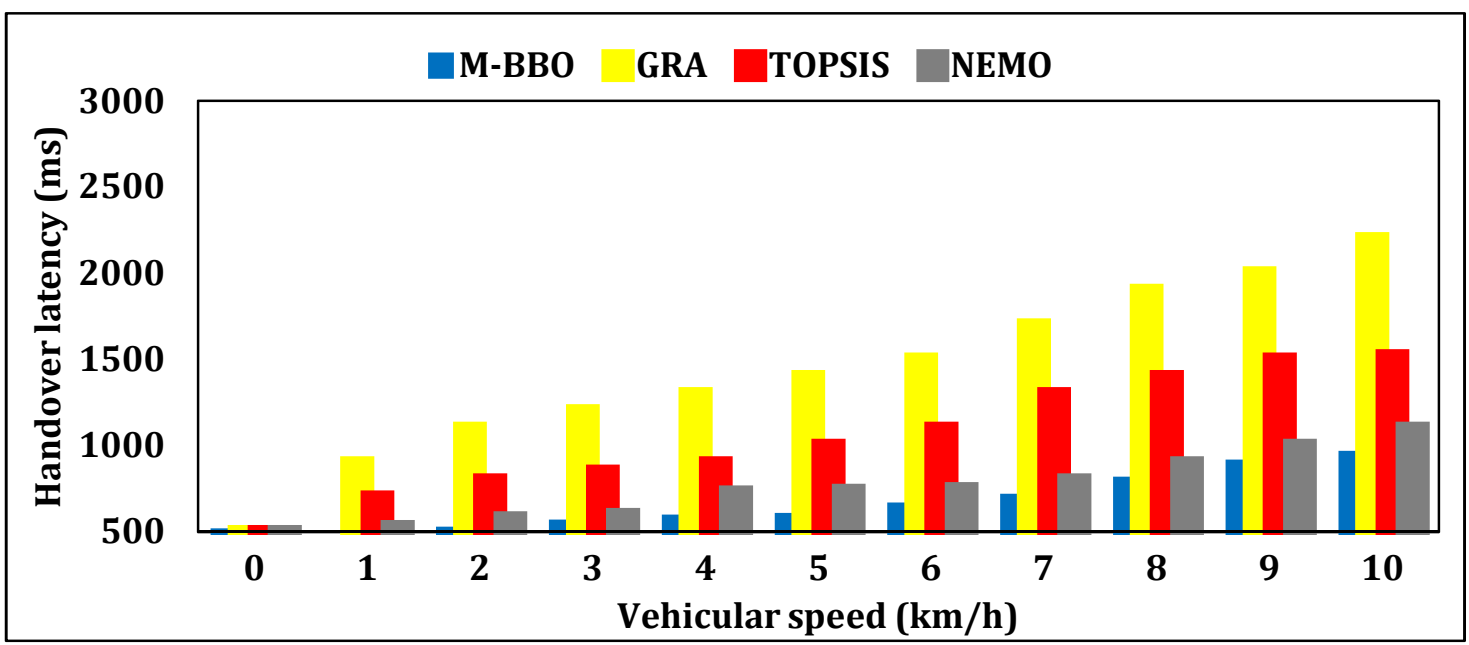

Figure 7 (e). Handover latency vs. vehicular speed

Figure 7(f) shows the impact of various vehicular densities to handover latency. The number of vehicles is adjusted between $(10-100)$. Per vehicle is moving at a fixed speed $(50 \mathrm{~km} / \mathrm{h})$. In place of the vehicle density rise, the handover latency also rises because density cause more congestions and the handover latency will be increased. The M-BBO and NEMO show better performance, followed by TOPSIS and GRA models.

The scheme for the selection of a network is according to different parameters namely jitter, delay, BER, loss of packets, cost of communication, time to respond, and network loading. A comparison is made in the scheme that is proposed and the TOPSIS and GRA decision models in the context of failed attempts at handovers, handovers that are frequent, ratio of packet loss, as well as the throughput. The proposed scheme outperforms in the area of minimizing the rate of handoff and in maximizing the throughput with the decision models of the GRA and TOPSIS. 


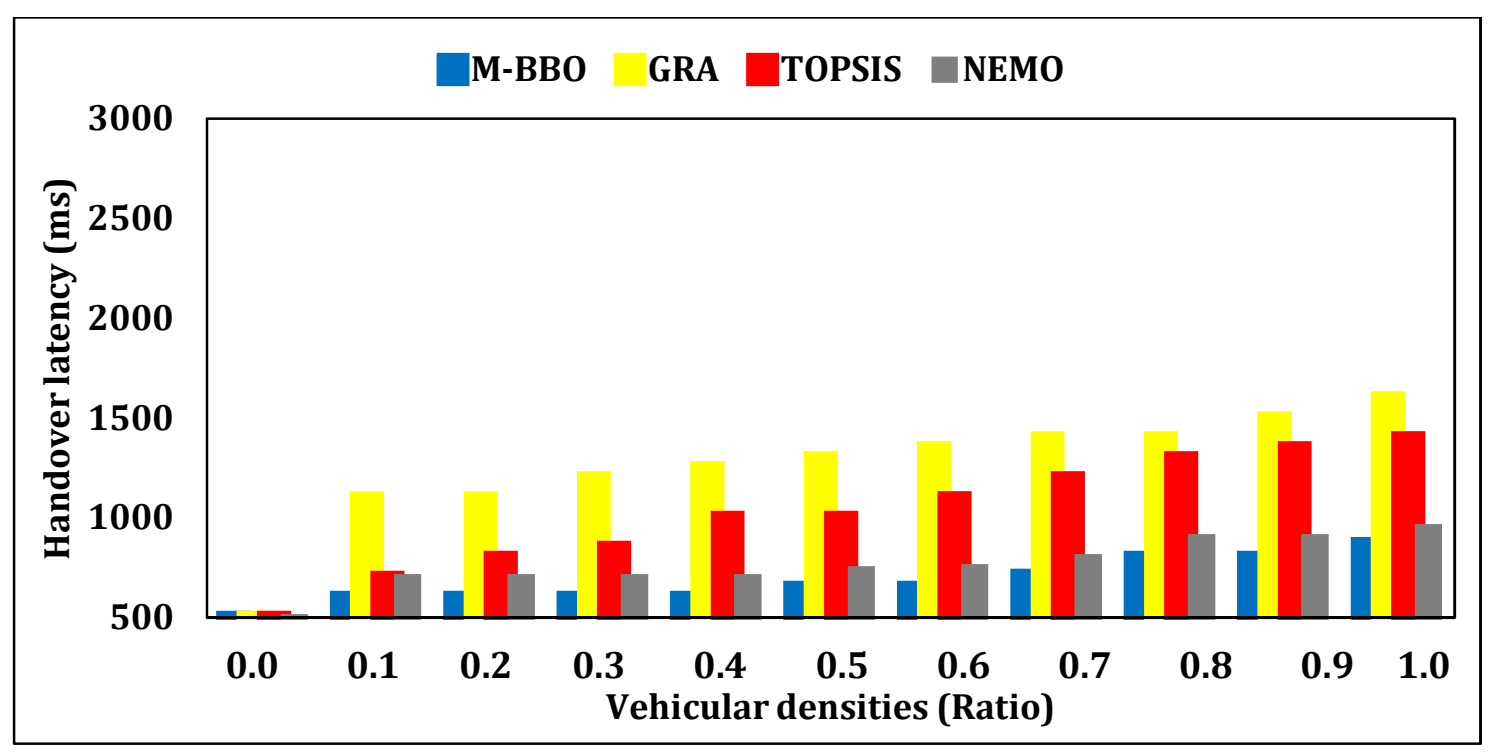

Figure 7 (f). Handover latency vs. vehicular densities

\section{Conclusions and Future work}

This study has proposed an algorithm for the vertical handover decision-making process known as the M-BBO. This algorithm chooses the best network candidate, which addresses the issues of requirements for connectivity, by considering the preferences of the user in the vehicular setting. In order to achieve this, the M-BBO makes use of the currently available feature of the OBUs including the GPS-based geo-navigation and geo-location, multiple interfaces of wireless networks, as well as the strong computing resources. In addition, the IEEE 802.21 standard offers services that assist in empowering the M-BBO. It was demonstrated throughout the simulation that the MBBO is capable of selecting the best network candidate accurately based on the requirements of the connection in accordance with the preferences of the user as well as the requirements of the application.

There are many directions needed to investigate to support the proposed architecture. The main could be a more appropriate mobility management and fog orchestration models that take $\mathrm{M}-\mathrm{BBO}$ characteristics into account to support fog computing.

\section{Acknowledgments}

The authors thank the Universiti Teknologi Malaysia (UTM), Malaysian Japan International Institute of Technology (MJIIT), and i-Kohza Computer System and 
Network (CSN). We also extend our sincere appreciation to the Deanship of Scientific

Research at King Saud University for funding this work through research Group number

(RG-288).

\section{References}

1. D. Miorandi, S. Sicari, F. De Pellegrini, and I. Chlamtac, "Internet of things: vision, applications and research challenges," AdHoc Networks, vol. 10, no. 7, pp. 1497-1516, 2012.

2. L. Atzori, A. Iera, and G. Morabito, "The internet of things: a survey," Computer Networks, vol. 54, no. 15 , pp. 2787-2805, 2010.

3. J. Gubbi, R. Buyya, S. Marusic, and M. Palaniswami, "Internet of Things (IoT): a vision, architectural elements, and future directions," Future Generation Computer Systems, vol. 29, no. 7,pp. 1645-1660, 2013.

4. P. S. Henry and H. Luo, "WiFi: what's next?" IEEE Communications Magazine, vol. 40, no. 12, pp. 66-72, 2002.

5. Broustis and M. Faloutsos, "Routing in vehicular networks: Feasibility, modeling, and security," Int. J. Veh. Technol., vol. 2008, pp. 1-8. Dec. 2008. [Online]. Available: http://www.hindawi.com/GetArticle. aspx?doi $=10.1155 / 2008 / 267513 \& \mathrm{e}=$ cta.

6. IEEE Standard for Local and Metropolitan Area Networks-Part 21: Me- dia Independent Handover, Jun. 21, 2009. [Online]. Available: http://dx. doi.org/10.1109/ ieeestd. 2009. 4769367.

7. Jiang, D., Xu, Z., \& Lv, Z. (2015). A multicast delivery approach with minimum energy consumption for wireless multi-hop networks. Telecommunication Systems, 62(4), 771-782.

8. Jiang, D., Xu, Z., Wang, W., Wang, Y., \& Han, Y. (2015). A collaborative multi-hop routing algorithm for maximum achievable rate. Journal of Network and Computer Applications, 57, 182-191.

9. Jiang, D., Wang, Y., Yao, C., \& Han, Y. (2015). An effective dynamic spectrum access algorithm for multi-hop cognitive wireless networks. Computer Networks, 84, 1-16.

10. Jiang, D., Xu, Z., Li, W., \& Chen, Z. (2015). Network coding-based energy-efficient multicast routing algorithm for multi-hop wireless networks. Journal of Systems and Software, 104, 152-165.

11. Akyildiz IF, McNair J, Ho JSM, Uzunalioglu H, Wang W. Mobility management in next-generation wireless systems.Proceedings of the IEEE 1999; 87(8):1347-1384.

12. Ernst T, Lach HY. Network Mobility Support Terminology. RFC 4885, July 2007.

13. J. Marquez-Barja, C. T. Calafate, J.-C. Cano, and P. Manzoni, "An overview of vertical handover techniques: Algorithms, protocols and tools,"Comput. Commun., vol. 34, no. 8, pp. 985-997, Jun. 2011. [Online]. Available: http://dx.doi.org/10.1016/j.comcom.2010.11.010

14. Petrut, I., Otesteanu, M., Balint, C., \& Budura, G. (2015, July). HetNet handover performance analysis based on RSRP vs. RSRQ triggers. In Telecommunications and Signal Processing (TSP), 2015 38th International Conference on (pp. 232-235). IEEE.

15.Zhang, H., Jiang, C., Cheng, J., \& Leung, V. C. (2015). Cooperative interference mitigation and handover management for heterogeneous cloud small cell networks. IEEE Wireless Communications, 22(3), 92-99.

16. W. D. Hsiao, Y. X. Liu, and H. C. Chao, "An intelligent WiMAX mobile network handoff mechanism with GPS consideration,” in Proc. Int. ACM Conf. Mobile Technol. Appl. Syst., 2008, pp. 1-5. [Online]. Available:http://dx.doi.org/10.1145/1506270.1506404

17. M. Ylianttila, J. Makela, and K. Pahlavan, "Analysis of handoff in a location-aware vertical multiaccess network," Comput. Netw.,vol. 47, no. 2, pp. 185-201, Feb. 2005. [Online]. Available: http://www.sciencedirect.com/science/article/pii/S138912860400194X

18. C. Gu, M. Song, Y. Zhang, and J. Song, "Access network selection strategy using position prediction in heterogeneous wireless networks," Frontiers Electr. Electron. Eng. China, vol. 5, no. 1, pp. 23-28, Mar. 2010. [Online]. Available: http://dx.doi.org/10.1007/s11460-009-0064-4.

19. Su, D., Wen, X., Zhang, H., \& Zheng, W. (2010, April). A self-optimizing mobility management scheme based on cell ID information in high velocity environment. In Computer and Network Technology (ICCNT), 2010 Second International Conference on (pp. 285-288). IEEE.

20. Wang, C. Fan, C. H. Hsu, Q. Sun, and F. Yang, "A vertical handoff method via self-selection decision 
tree for internet of vehicles,"IEEE Syst. J., to be published. [Online]. Available: http://dx.doi.org/ 10.1109 /jsyst.2014.2306210

21. L.-C. Wang, A. Chen, and A. Chen, "Network selection with joint vertical and horizontal handoff in heterogeneous WLAN and mobile WiMax systems," in Proc. IEEE 65th Veh. Technol. Conf., Apr. 2007, pp. 794-798.[Online]. Available: http://dx.doi.org/10.1109/vetecs.2007.173

22. B. Ma and X. Liao, "Speed-adaptive vertical handoff algorithm based on fuzzy logic in vehicular heterogeneous networks," in Proc. 9th Int.Conf. Fuzzy Syst. Knowl. Discovery, May 2012, pp. 371375. [Online].Available: http://dx.doi.org/10.1109/fskd.2012.6234358

23. Y. Tao and R. Peng, "A fuzzy logic vertical handoff algorithm with motion trend decision," in Proc. 6th Int. Forum Strategic Technol., Aug. 2011,pp. 1280-1283. [Online]. Available: http://dx.doi.org/10.1109/ifost.2011.6021253

24. J. D. Martinez-Morales, U. Pineda-Rico, and E. Stevens-Navarro, "Performance comparison between MADM algorithms for vertical handoff in 4G networks," in Proc. 7th Int. Conf. CCE, Sep. 2010, pp. 309-314. [Online].Available: http://dx.doi.org/10.1109/iceee.2010.5608646

25. A. Ismail and B.-H. Roh, "Adaptive handovers in heterogeneous networks using fuzzy MADM," in Proc. ICMIC, Sep. 2011, pp. 99- 104.

26. K. Shafiee, A. Attar, and V. C. M. Leung, "Optimal distributed vertical handoff strategies in vehicular heterogeneous networks," IEEE J. Sel.Areas Commun., vol. 29, no. 3, pp. 534-544, Mar. 2011. [Online].Available: http://dx.doi.org/10.1109/jsac.2011.110304.

27. Zhang, H., Hui, L. I. U., Wenmin, M. A., Zheng, W., Xiangming, W. E. N., \& Jiang, C. (2012). Mobility robustness optimization in femtocell networks based on ant colony algorithm. IEICE transactions on communications, 95(4), 1455-1458.

28. Goudarzi, S., Hassan, W. H., Anisi, M. H., Soleymani, S. A., \& Shabanzadeh, P. (2015). A Novel Model on Curve Fitting and Particle Swarm Optimization for Vertical Handover in Heterogeneous Wireless Networks. Mathematical Problems in Engineering, 2015.

29. Sangeetha, S., \& Victoire, T. A. A. (2016). Radio Access Technology Selection in Heterogeneous Wireless Networks Using a Hybrid Fuzzy-Biogeography Based Optimization Technique. Wireless Personal Communications, 87(2), 399-417.

30. Pacheco-Paramo, D., Pla, V., Casares-Giner, V., \& Martinez-Bauset, J. (2012). Optimal radio access technology selection on heterogeneous networks. Physical Communication, 5(3), 253-271.

31. Carvalho, G. H., Woungang, I., Anpalagan, A., Coutinho, R. W., \& Costa, J. C. (2013). A semiMarkov decision process-based joint call admission control for inter-RAT cell re-selection in next generation wireless networks. Computer Networks, 57(17), 3545-3562.

32. El Helou, M., Ibrahim, M., Lahoud, S., \& Khawam, K. (2014). Optimizing network information for radio access technology selection. In IEEE symposium on computers and Communication (ISCC) (pp. $1-6)$.

33. El Helou, M., Ibrahim, M., Lahoud, S., \& Khawam, K. (2013). Radio access selection approaches in heterogeneous wireless networks. In IEEE 9th international conference on wireless and mobile computing, networking and communications (WiMob) (pp. 521-528).

34. V. Devarapalli, R. Wakikawa, A. Petrescu, and P. Thubert, "Network mobility (NEMO) basic support protocol," Internet Engineering Task Force (IETF), vol. RFC-3963, pp. 1-33, 2005.

35. Chen, Y. S., Hsu, C. S., \& Cheng, C. H. (2014). Network mobility protocol for vehicular ad hoc networks. International Journal of Communication Systems, 27(11), 3042-3063.

36. Simon, D. (2008). Biogeography-based optimization. IEEE transactions on evolutionary computation, 12(6), 702-713.

37. Goudarzi, S., Hassan, W. H., Anisi, M. H., \& Soleymani, S. A. (2017) MDP-Based Network Selection Scheme by Genetic Algorithm and Simulated Annealing for Vertical-Handover in Heterogeneous Wireless Networks. Wireless Personal Communications, 92 (2), 399-436.

38. Saaty, T. L. (1988). What is the analytic hierarchy process? In Mathematical models for decision support (pp. 109-121). Springer Berlin Heidelberg.

39. Hwang CL, Yoon K (1981) Multiple attributes decision making methods and applications. Springer, Berlin.

40. Kuo, Yiyo, Yang, Taho, \& Huang, Guan-Wei. (2008). The use of grey relational analysis in solving multiple attribute decision-making problems. Computers \& Industrial Engineering, 55(1), 80-93.

41. Wang, Y., Yuan, J., Zhou, Y., Li, G., \& Zhang, P. (2008, March). Vertical handover decision in an enhanced media independent handover framework. In 2008 IEEE Wireless Communications and 
Networking Conference (pp. 2693-2698). IEEE.

42. V H. Ma and D. Simon, "Blended biogeography-based optimization for constrained optimization," Engineering Applications of Artificial Intelligence, vol. 24, no. 3, pp. 517 - 525, 2011. [Online]. Available:http://www.sciencedirect.com/science/article/pii/S0952197610001600.

43. H. Mo and L. Xu, "Biogeography based optimization for traveling salesman problem," in Natural Computation (ICNC), 2010 Sixth International Conference on, vol. 6, Aug. 2010, pp. $3143-3147$, doi:10.1109/ICNC.2010.5584489.

44. Y. Song, M. Liu, and Z. Wang, "Biogeography-based optimization for the traveling salesman problems," in Computational Science and Optimization (CSO), 2010 Third International Joint Conference on, vol. 1, May 2010, pp. 295 -299, doi: 10.1109/CSO.2010.79

45. V. K. Panchal, Parminder Singh, Navdeep Kaur, Harish Kundra, "Biogeography based satellite image classification," International Journal of Computer Science and Information Security, vol. 6, pp. 269274, 2009.

46. Singh, S., \& Mittal, E. (2013, April). Range based wireless sensor node localization using PSO and $\mathrm{BBO}$ and its variants. In Communication Systems and Network Technologies (CSNT), 2013 International Conference on (pp. 309-315). IEEE.

47. Stevens-Navarro, E., Lin, Y., \& Wong, V. W. (2008). An MDP-based vertical handoff decision algorithm for heterogeneous wireless networks. IEEE Transactions on Vehicular Technology, 57(2), 1243-1254.

48. C. Guo, Z. Guo, Q. Zhang, and W. Zhu, "A Seamless and Proactive Endto-End Mobility Solution for Roaming Across Heterogeneous Wireless Networks," IEEE Journal on Selected Areas in Communications, vol. 22,no. 5, pp. 834-848, June 2004.

49. O. Ormond, J. Murphy, and G. Muntean, "Utility-based Intelligent Network Selection in Beyond 3G Systems," in Proc. of IEEE ICC'06, Istanbul, Turkey, June 2006. Navarro E, Wong W, Xia L. Comparision between vertical handoff decision algorithms for heterogeneous wireless networks. IEEE Vehicular Technol Conf 2006:947-51.

50. Ormond, Olga, Perry, Philip, \& Murphy, John. (2005). Network selection decision in wireless heterogeneous networks. Paper presented at the 2005 IEEE 16th International Symposium on Personal, Indoor and Mobile Radio Communications.

51. Stevens-Navarro, E., \& Wong, V. W. (2006, May). Comparison between vertical handoff decision algorithms for heterogeneous wireless networks. In 2006 IEEE 63rd Vehicular Technology Conference (Vol. 2, pp. 947-951). IEEE.

52. H. Ma, D. Simon, M. Fei, and Z. Chen, "On the equivalences and differences of evolutionary algorithms," Eng. Appl. Artif. Intell., vol. 26,no. 10, pp. 2397-2407, 2013.

53. J. Derrac, S. Garcia, D. Molina, and F. Herrera, "A practical tutorial on the use of nonparametric statistical tests as a methodology for comparing evolutionary and swarm intelligence algorithms," Swarm Evol. Comput, vol. 1, no. 1, pp. 3-18, 2011.

54. P. N. Suganthan. (Feb. 2011). CEC11 Competition on Testing Evolutionary Algorithms on Real-World Numerical Optimization Problems. [Online]. Available: http://www3.ntu.edu.sg/ home/EPNSugan/

55. Marquez-Barja, J. M., Ahmadi, H., Tornell, S. M., Calafate, C. T., Cano, J. C., Manzoni, P., \& DaSilva, L. A. (2015). Breaking the vehicular wireless communications barriers: Vertical handover techniques for heterogeneous networks. IEEE Transactions on Vehicular Technology, 64(12), 5878-5890. 